\title{
Autonomic nervous system and inflammation interaction in endometriosis- associated pain
}

\author{
Yajing Wei ${ }^{1 \dagger}$, Yanchun Liang ${ }^{1 \dagger}$, Haishan Lin $^{2}$, Yujing Dai ${ }^{2}$ and Shuzhong Yao ${ }^{1 *}$ (D)
}

\begin{abstract}
Endometriosis is a chronic inflammatory disease. Pain is the most common symptom in endometriosis. Endometriosis-associated pain is caused by inflammation, and is related to aberrant innervation. Although the specific mechanism between endometriosis-associated pain and the interaction of aberrant innervation and inflammation remains unclear, many studies have confirmed certain correlations between them. In addition, we found that some chronic inflammatory autoimmune diseases (AIDs) such as inflammatory bowel disease (IBD) and rheumatoid arthritis (RA) share similar characteristics: the changes in dysregulation of inflammatory factors as well as the function and innervation of the autonomic nervous system (ANS). The mechanisms underlying the interaction between the ANS and inflammation have provided new advances among these disorders. Therefore, the purpose of this review is to compare the changes in inflammation and ANS in endometriosis, IBD, and RA; and to explore the role and possible mechanism of sympathetic and parasympathetic nerves in endometriosis-associated inflammation by referring to IBD and RA studies to provide some reference for further endometriosis research and treatment.
\end{abstract}

Keywords: Endometriosis, Autonomic nervous system, Inflammation, Pain

\section{Background}

Endometriosis is an estrogen-dependent benign gynecological disease. Approximately 10\% of reproductive-age women are affected by endometriosis worldwide. This disease is characterized by the presence of ectopic endometrial tissue outside of the uterine cavity $[1,2]$. Ectopic endometrial tissues consist of glandular and stromal cells, macrophages, nerves, and blood vessels [3]. Even if the pathogenesis is unclear, endometriosis is certainly a chronic inflammation disorder [4]. The levels and concentrations of active macrophages; interleukin (IL)-1 $\beta$, IL-6, IL-8; nerve growth factor (NGF); other immune cells; and inflammatory

\footnotetext{
*Correspondence: yszzlfy@163.com

${ }^{\dagger}$ Yajing Wei and Yanchun Liang contributed equally to this work. 'Department of Obstetrics and Gynecology, First Affiliated Hospital of Sun Yat-Sen University, No. 58, the 2nd Zhongshan Road, Yuexiu District, Guangzhou 510080, Guangdong, China

Full list of author information is available at the end of the article
}

factors are increased in peritoneal fluid (PF) and endometriotic lesions [4-6]. These changes are believed to contribute to serious symptoms of pain such as chronic pelvic pain, dysmenorrhea, and dyspareunia [7]. Notably in deep infiltrating endometriosis (DIE) and intestinal endometriosis, the anatomical distribution of lesions is normally more closely related to pelvic pain symptoms [2]. Abnormal innervations are observed in most endometriotic lesions: an increased number of total intact nerve fibers, increased sensory and decreased sympathetic nerve fiber density (NFD) [6], the occurrence of cholinergic and unmyelinated nerve fibers, etc. [8] In various studies, these abnormal phenomena have been correlated with endometriosisassociated pain $[6,8-10]$. More importantly, sympathetic and parasympathetic systems have different inflammation-related effects in different stages of 
inflammation [10]. Many researchers have found that the function and innervation of the autonomic nervous system (ANS) are altered in chronic inflammatory AIDs [6], such as Crohn's disease (CD) [11]. However, whether the inflammation induced by abnormal sympathetic and parasympathetic innervation has any effect on endometriotic lesions is unclear. The purpose of this review is to elaborate on the effects of sympathetic and parasympathetic nerve fibers on endometriosis-associated inflammation and to explain the underlying mechanism of action.

\section{Endometriosis and inflammation}

Endometriosis is considered a chronic inflammatory disorder. There are a series of alterations of inflammatory cells, cytokines, and chemokines in endometriotic lesions and PF, forming an inflammatory microenvironment. More importantly, the inflammatory niche also interacts with endometriotic cells (including stromal cells and epithelial cells), which plays an important role in the development and maintenance of endometriosis.

Menstruation is an inflammatory process characterized by an increase in a variety of tissue-resident immune cells. A complex interaction between resident immune cells and uterine stromal cells modulates the biosynthesis and release of pro-inflammatory cytokines, chemokines, and prostaglandins (PGs), resulting in local vasoconstriction [12]. Menstrual materials retrograde flow to the peritoneal cavity and implant into tissues [13]. During lesion formation, inflammatory cells are recruited to the lesions. The recruited inflammatory cells secrete multiple inflammatory factors. Macrophages secrete and promote the release of IL-1 family factors (including IL-1 $\beta$, IL-37, etc.), IL-6, and tumor necrosis factor- $\alpha$ (TNF- $\alpha)[7,8,14]$. Mast cells (MCs) release IL-2, IL-3, IL-6, IL-7, IL-9, IL-10, IL-25, and NGF, etc. [5, 7, 10] Neutrophils release IL-8, IL-17, and IL-17 $\alpha$ [15-17]. Furthermore, other inflammatory cells secrete factors such as IL-33, MCP1, IL-10, and IL-4. Moreover, endometriotic lesions can induce the expression of PGs, MCP1, glycodelin, and other inflammatory mediators and pain-associated substances $[10,18,19]$. Specifically, PGE2, PGF2 $\alpha$, and TNF- $\alpha$ are produced and increased in the early stage; TNF- $\alpha$, NGF, and IL-17 can cause persistent inflammation; PGE2, PGF2 $\alpha$, transforming growth factor- $\beta$ (TGF$\beta$ ), glycodelin, and TNF- $\alpha$ can induce the sensation of pain $[3,10,20-22]$. These inflammation-associated cytokines, chemokines, other inflammatory mediators, and painassociated substances act on inflammatory cells in turn. These retroactions lead to more inflammatory cell recruitment in lesions. These substances alter the original environment of peritoneal and pelvic environments and form a new inflammatory microenvironment. The growth, implantation, infiltration, and migration of endometriosis lesions occur subsequently and retroact on inflammatory cells and substances. This vicious cycle contributes to aggregation of the endometriosis-associated inflammation (Fig. 1).

Katherine A Burns et al. suggested that endometriosis is divided into two stages (immune-predominant phase and hormone-predominant phase). They have demonstrated that early stage $(<72 \mathrm{~h})$ of endometriosis is predominantly dependent on the signaling of the innate immune system, whereas estradiol/estrogen receptor $\alpha /$ IL-6(E2/ER $\alpha / \mathrm{IL}-6)$-mediated cross-talk plays a partial role. In this stage of endometriosis animal models, inflammatory factors and vascular endothelial growth factor (VEGF) are increased in mRNA and protein levels, but they are independent of estradiol (E2) treatment. In addition, inflammatory cells are recruited to peritoneal cavity via disease-dependent way regardless of ER. They find that early initiation phase of endometriosis $(<72 \mathrm{~h})$ is largely regulated by the innate immune system. Immune system signaling predominates E2-mediated signaling in disease at this stage. According to their findings, they suggest that there are two phases of endometriosis: immunepredominant phase and hormone-predominant phase. As a result, targeting the innate immune system could prevent lesion attachment in this susceptible population of women [23].

\section{Inflammatory cells Macrophages}

Macrophages, an important member of the immune system, are activated mononuclear phagocytes recruited in endometriotic lesions [14, 24, 25]. Macrophages are classically divided into two typical phenotypes: M1 macrophages (classical activated macrophages) and M2 macrophages (alternatively activated macrophages). M1 macrophages are activated by interferon (IFN)- $\gamma$, TNF$\alpha$, and lipopolysaccharide. These cells can produce proinflammatory cytokines and chemokines that participate in the early stage of injury, pro-inflammatory response, and myoblast proliferation. M2 macrophages are activated by IL-4, IL-10, IL-13, and TGF- $\beta$. Once activated, M2 macrophages secrete anti-inflammatory cytokines, growth factors, and other reparative factors, which are involved in the anti-inflammatory response and advanced stage of the repair and healing process $[14,24,26,27]$. The bidirectional differentiation of total macrophages is caused by different polarizing tendencies of large peritoneal macrophages (LPMs) and small peritoneal macrophages (SPMs). LPMs are involved in an M1 polarization trend and are major contributors to the M1 polarization of total macrophages. Moreover, the tendency of M2 polarization of SPMs plays a major role in M2 differentiation of total macrophages, which appears earlier than the emergence of M1 macrophages [28]. 


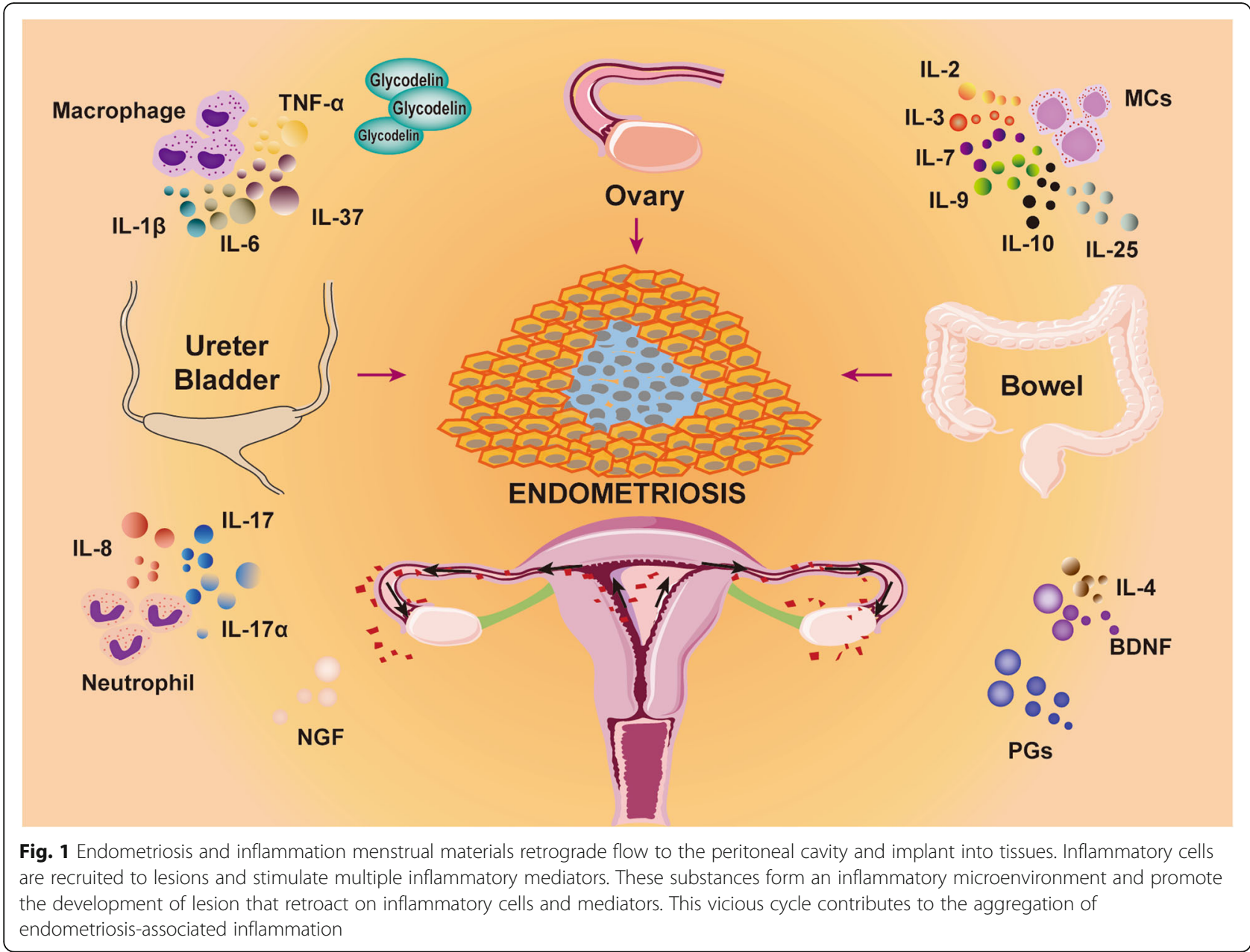

Macrophages are recruited to the peritoneum and lesions of endometriosis. This process usually occurs in the sites of hypoxia and tissue stress, where they clear cell debris, heme-iron, and generate proliferation and pro-angiogenesis signals. In murine, macrophages are required for lesion establishment and growth. Bone marrow-derived Tie-2expressing macrophages specifically contribute to neovascularization in lesions, possibly because they are associated with the recruitment of circulating endothelial progenitors, and sustain their survival as well as the integrity of the vessel wall [24]. After recruitment to the peritoneal cavity, macrophages mainly present an M2 phenotype, accounting for $80 \%$ of lymphocytes in the PF in human endometriosis and mouse endometriosis models $[15,29]$. In a mouse model, endogenous macrophages are reportedly involved in tissue remodeling during the development of endometriosis, and the M1 to M2 phenotypic transition is required for the growth of ectopic lesions [30]. Furthermore, transplantation of bone marrow-derived M2 macrophages promotes the development of endometriosis in Balb/C murine models. In the murine endometriosis model, flow cytometry analysis demonstrated that the proportion of LPMs $\left(\mathrm{F} 4 / 80^{\text {high }} \mathrm{CD} 11 \mathrm{~b}^{\text {high }}\right.$ cells) in the peritoneal cavity decreased immediately with the injection of peritoneal endometrial injection, and the reduced percentage of LPMs persisted until 42 days after injection. It can be proposed that LPMs play a crucial role in the early survival of recurrent endometrial tissues. Then, SPMs participate in complex reciprocities in the pathogenesis of endometriosis [28]. These results suggest that M2 macrophages play an important role in endometriotic lesion establishment and development.

Peritoneal macrophages from patients with endometriosis display increased activation of the pro-inflammatory transcription factor NF- $\mathrm{kB}$ and enhanced protein expression of pro-inflammatory cytokines such as TNF- $\alpha$, IL- 6 , IL- $1 \beta$, and TGF- $\beta[1,5,8]$. NF- $\kappa B$, a central regulator of gene expression in the immune system, plays a regulatory role in reproductive tissues. IL- 6 is under the control of NF- $\kappa$ B. The reduced levels of IL- 6 are observed exclusively at the late secretory phase in eutopic endometrium. High serum IL-6 concentrations in endometriosis patients are probably related to the reduction in 
conserved helix-loop-helix ubiquitous kinase (CHUK) protein as well as NF- $\mathrm{KB}$ inhibitor alpha (NFKBIA) mRNA and the p-NFKBIA/NFKBIA protein ratio. Both proteins are involved in the mechanism of NF- $\mathrm{KB}$ activation that may affect RELA nuclear location, RELA nuclear/cytoplasmic ratio, and NF- $\mathrm{kB}$ DNA binding. The milieus of pro-inflammatory factors create a microenvironment that encourages endometrial cell attachment, invasion, and angiogenesis. In this microenvironment, cell proliferation is enhanced and more inflammatory signaling is activated [31, 32].

Macrophages are abundantly recruited to lesions of endometriosis after activation by certain chemokines and cytokines. These cells can release different types of inflammatory substances, creating an inflammatory microenvironment that contributes to the establishment and growth of endometriotic lesions. In turn, these changes can induce the recruitment of macrophages and as a result, form a vicious circle during the development of endometriosis.

\section{Mast cells}

In addition to macrophages, the numbers of mast cells (MCs) and activated MCs are clearly increased in human or animal endometriosis [33, 34]. Compared to other sites, DIE lesions have a significantly greater number of MCs, activated MCs and degranulating MCs [8]. MCs are critical sentinel cells in innate and adaptive immune systems. These cells are present in all tissues and are particularly abundant in tissues and mucous membranes that can respond to external inflammatory stimuli and pathogens [35]. MCs are best known for the generation and release of mediators of allergic reactions [35]. Previous studies have indicated a higher prevalence of allergic disease among endometriosis patients. Th2 cells and MCs express IL-25, which is increased in the PF of patients with endometriosis [36]. Moreover, MCs can produce growth factors, costimulatory molecules, and numerous pro- and anti-inflammatory mediators [35, 37, 38]. When activated, MCs release inflammatory mediators from their storage granules as well as via phospholipid membrane metabolism after de novo synthesis of cytokines and chemokines [39]. These mediators include IL-2, IL-3, IL-6, IL-7, IL-9, IL-10, IFN- $\gamma$, TNF- $\alpha$, and chemokines (CXCL8, CCL2, and CCL5) [38]. The activation of MCs is important for proper defense against certain helminth infections and other parasites. However, MCs are potentially lethal cells on widespread activation, which occurs in fatal anaphylaxis, and can cause tissue damage with sustained activation, as in chronic inflammation [35].

RBL2H3 cell (a rat basophilic leukemia cell line, a mucosal mast cell analog) degranulation is increased after E2 treatment [40]. The activation of RBL2H3 cells by E2 triggers the release of biologically active NGF, which promotes neurite outgrowth in PC12 cells and sensitizes dorsal root ganglion cells via the upregulation of Nav1.8 and transient receptor potential cation channel (subfamily $\mathrm{V}$ member 1) expression levels. This phenomenon is correlated with endometriosis-related dysmenorrhea [40]. Additionally, activated MCs contribute directly to neuropathic pain symptoms by releasing mediators such as histamine, leukotrienes, tryptase, TNF- $\alpha$, PGs, serotonin, IL-1, and IL-8. The activation of MCs may also contribute indirectly to the development of neuropathic pain by recruiting leukocytes that release algesic mediators [33]. As mentioned above, IL-25 is expressed by Th2 cells and MCs. The increased level of IL-25 can in turn stimulate IgE production, exacerbating a Th2 response. This process favors the development of allergies by perpetuating a hypersensitivity reaction in endometriosis patients [36].

Both activation and degranulation of MCs have been widely found in endometriotic lesions in animal models and humans. These processes can maintain the state of chronic inflammation and allergic reactions in endometriosis through releasing cytokines and chemokines.

\section{Neutrophils}

The infiltration of neutrophils into the peritoneal cavity is significantly increased in women with endometriosis compared with that in healthy women, especially in the early stage of endometriosis [23, 41, 42]. In a mouse endometriosis model, neutrophil infiltration in ectopic uterine tissue peaked on days 1-5 and subsequently declined on day 6 or 7 , suggesting an important role for neutrophils in the early stages of lesion development [1]. Neutrophils are considered simple foot soldiers of the innate immune system. Neutrophils are undoubtedly major effectors of acute inflammation, and several lines of evidence indicate that they also contribute to chronic inflammatory conditions as well as adaptive immune responses [43]. In endometriosis, neutrophils in the abdominal cavity can secrete an effective pro-angiogenic factor, VEGF, which is also increased in the PF in the endometriosis. As a result, neutrophils may support the growth of endometriosis lesions by secreting VEGF. Moreover, there may be some other nonclassical factors secreted by neutrophils that can promote inflammation and neovascularization in endometriosis [42, 44]. In addition, neutrophils can produce IL-17 $\alpha$ which is increased in endometriosis patients and associated with severity and infertility of endometriosis [16, 45-47].

In brief, neutrophils are increased mainly in the early stage of endometriosis and may be correlated with the early development of endometriotic lesions. Neutrophils may affect the occurrence and development of endometriotic lesions through the inflammatory-immune pathway and promote angiogenesis in lesions (Fig. 2). 


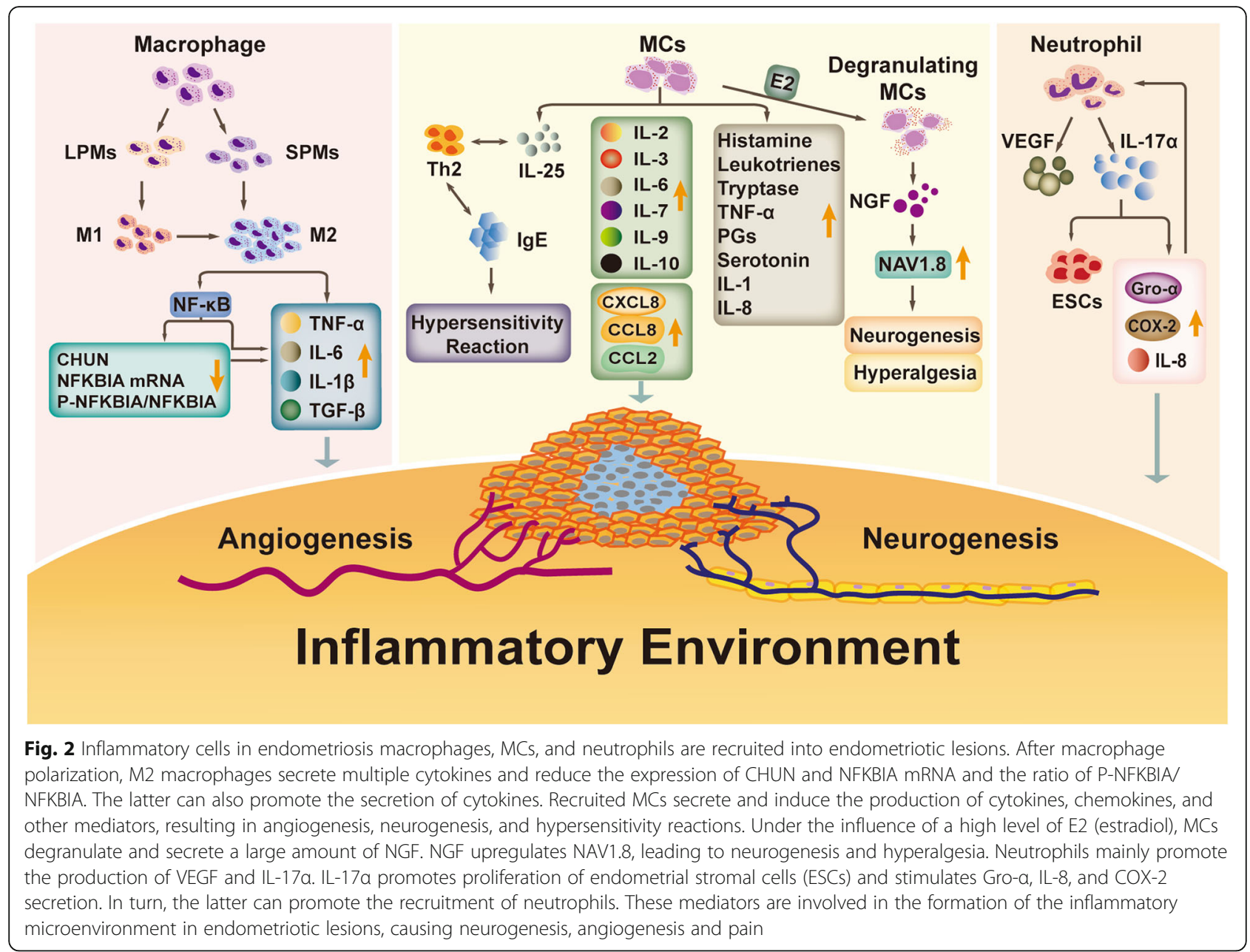

\section{Inflammatory mediators}

The growth of endometriotic lesions stimulates the production and secretion of pro-inflammatory cytokines, chemokines, and growth factors [3], suggesting that relative inflammatory mediators play a vital role in the development of endometriosis.

\section{Interleukins}

The largest family of interleukins is the IL- 1 family. The IL-1 is a central mediator of innate immunity and inflammation. IL-1 family includes seven ligands with agonist activity (IL-1, IL-1 $\beta$, IL-18, IL-33, IL-36 $\alpha$, IL36 $\beta$, and IL36y), three receptor antagonists (IL-1R $\alpha$, IL-36R $\alpha$, and IL-38), and an anti-inflammatory cytokine (IL-37) [48]. IL-1 is produced mainly by monocytes and macrophages. The concentration level of IL-1 is increased in the PF, serum, and lesions of endometriosis patients. The imbalance between IL- $1 \alpha$, pro-IL-1 $\beta$, sIL-1R2, and sIL-1RAcP in the PF and serum of endometriosis patents may be linked to the ability to transform acute inflammation into a chronic one [49]. IL-1 3 , which reportedly exhibits an excessive concentration and activity in endometriosis, is a potent pro-inflammatory cytokine synthesized by macrophages. IL- $1 \beta$ stimulates the production of eutopic endometrial stromal cells (ESCs) brain-derived neurotrophic factor (BDNF) at the mRNA and protein levels in an IL-1 receptor-dependent fashion by c-Jun $\mathrm{N}$-terminal kinase (JNK), NF- $\mathrm{BB}$, and mechanistic target of rapamycin signal transduction pathways. This process can aggravate endometriosis-associated pain and inflammation [5].

IL-37 is overexpressed in ovarian endometriosis patients $[50,51]$. The level of IL-37 reportedly correlates with the severity of endometriosis [52]. IL-37, a novel anti-inflammatory cytokine of the IL-1 family, is generated by various types of immune cells. Normally, IL-37 is present in inflammatory processes and acts as an inhibitor of the inflammatory response. IL-37 binds to IL-18 receptor alpha and delivers the inhibitory signal by binding to TIR8. Moreover, IL-37 can be protective in inflammation and injury as well as inhibit both innate and adaptive immunity [50-53]. This theory can be demonstrated in an endometriosis mouse model [51]. IL-37 synthesis is enhanced by IL- $1 \beta$, TNF- $\alpha$, IFN- $\gamma$, and 
TGF- $\beta$ in endometriosis. However, IL-37 overexpression in turn significantly suppresses both protein and mRNA expression of inflammatory cytokines (IL-1 $\beta$, IL-6, IL-10, TNF- $\alpha$, etc.) in cultured endometrial stromal cells. Moreover, a significant inverse correlation was observed between IL-37 mRNA and NF- $\mathrm{kB}$ mRNA expression [52]. However, the potential role of IL-37 in the pathogenesis of endometriosis has not been extensively investigated [51]. In other inflammatory AIDs, such as inflammatory bowel disease (IBD), the levels of IL-37 are also increased. Investigations have demonstrated that IL37 expression could be induced by TNF- $\alpha$ through activation of NF- $\mathrm{kB}$ and AP-1. Similarly, IL-37 can be upregulated by cytokines IL- $1 \beta$ and IL-10, reflecting that these cytokines have positive feedback effects on upregulating IL-37 production in inflammatory AIDs. These similar findings suggest a relationship between endometriosis and IBD. The findings of IBD may explain the changes in IL-37 and other inflammatory factors in endometriosis [54, 55].

Other interleukins are also altered in endometriosis patients. The increased IL-8 in the lesions of endometriosis stimulates cell proliferation and has a role in endometriosis as an autocrine regulator of endometrial cell growth. IL-8 has also been implicated as a prominent cytokine involved in angiogenesis $[17,56,57]$. Cell adhesion, transforming growth factor $\beta 1$ (TGF- $\beta 1$ ), and the pro-inflammatory cytokines IL- $1 \beta$ or TNF- $\alpha$ can synergistically promote IL-8 and VEGF expression in ESCs via the p38/ERK1/2 signaling pathways. The high levels of IL-8 and VEGF in the supernatant of ESCs stimulate the angiogenesis of human umbilical vein endothelial cells [58]. In addition, IL-8 exerts chemotactic activity primarily on neutrophils. These roles of IL-8 participate in the pathogenesis of endometriosis and may lead to transformation from acute to chronic inflammation [17, 56]. The levels of IL-25 and IL-17 $\alpha$ are also elevated in women with endometriosis. IL-17 $\alpha$, produced by neutrophils [16] or Th17 cells [59], is associated with severity and infertility of endometriosis [16, 45-47]. IL-17 $\alpha$ promotes proliferation of ESCs, and stimulates Gro- $\alpha$, IL-8, and COX2 secretion, recruiting more neutrophils and perpetuating inflammation in endometriosis $[16,47,60]$. The expression of the anti-inflammatory factor IL-10 is enhanced in endometriosis. IL-27 triggers IL-10 production in Th17 cells via c-Maf/RORyt/Blimp-1 signaling to promote the rapid growth and implantation of ectopic lesions [18]. The levels of IL-4 and IL-6 are increased remarkably in women with endometriosis $[19,61,62]$. IL6 levels are negatively correlated with the total annexin A1 (a pro-resolving and anti-inflammatory mediator) and formyl peptide receptor 2/aspirin-triggered lipoxin expression, suggesting that the reduction in resolution mediators may be responsible for the inflammatory process perpetuation as well as the maintenance and worsening of endometriosis [62].

\section{Transforming growth factor $\beta 1$}

TGF- $\beta 1$ is a pro-inflammatory factor and contributes to inflammatory pain and hyperalgesia $[10,63]$. TGF- $\beta 1$ has been demonstrated to be elevated in the PF of peritoneal endometriosis and DIE. This increased expression is correlated with the severity of dysmenorrhea $[8,10]$. TGF- $\beta 1$ regulates angiogenesis and inflammation in human endometriosis, which in turn provides a favorable microenvironment to attach floating uterine remnants at ectopic sites. TGF- $\beta 1$ promotes the activity and expression of RHOGTPases (an enzyme that is crucial for cell migration, cytoskeleton dynamics, adhesion, and inflammation) in ectopic endometrial tissues. A $\alpha 2-6$ sialylation is induced by TGF- $\beta 1$ through activating TGF- $\beta$ RI/ SMAD2/3 signaling in endometrial cells. In addition, TGF- $\beta 1$ can activate the P38MAPK molecular target to promote pro-inflammatory cytokines and proteolytic factors. All of the above findings support the important role of TGF- $\beta 1$ in the pathogenesis of endometriosis $[63,64]$.

\section{Tumor necrosis factor- $a$}

Concentrations of tumor necrosis factor- $\alpha$ (TNF- $\alpha$ ) are higher in PF and serum of endometriosis patients, especially in early stages of the disease [36, 65]. Increased TNF- $\alpha$ induces the expression of CXCL16 in eutopic ESCs, which enhances the function of the CXCL16/ CXCR6 axis. TNF- $\alpha$ may be associated with the increased motility of eutopic ESCs through the regulation of ERK1/ 2 signaling. As a result, TNF- $\alpha$ activates systemic and local inflammatory mechanisms in endometriosis development and progression, including elevated levels of chemokines and pro-inflammatory cytokines [66].

\section{Prostaglandin}

Prostaglandin (PG) is an important mediator of chronic inflammation [20]. The levels of PGE2 and PGF2 $\alpha$ are higher in endometriosis and adenomyosis patients and are positively correlated with the severity of vaginal hyperalgesia and dysmenorrhea, respectively [21, 65]. Traditionally, PGs have been thought to function mostly as mediators of acute inflammation. However, recent studies have demonstrated that in addition to their short-lived actions in acute inflammation, PGs can interact with cytokines and amplify the cytokine actions on various types of inflammatory cells, driving pathogenic conversion of these cells by critically regulating their gene expression. One mode of such PG-mediated amplification is to induce the expression of relevant cytokine receptors, which is typically observed in Th1 cell differentiation and Th17 cell expansion. The process of this mode can cause chronic immune inflammation. Another 
mode of amplification is cooperation of PGs with cytokines at the transcription level to activate NF- $\mathrm{BB}$ to induce the expression of inflammation-related genes. This signaling consequently enhances the expression of various NF- $\mathrm{kB}$-induced genes, including chemokines to macrophages and neutrophils, which enables sustained infiltration of these cells and further amplifies chronic inflammation [67].

\section{Noninflammatory factors}

In addition to inflammatory factors, noninflammatory factors are associated with endometriosis-associated inflammation in direct or indirect ways. NGF is a neurotrophic factor [68] but is also elevated in lesions of endometriosis. NGF expression is significantly higher in invasive lesions than in noninvasive lesions [22]. Some inflammatory cells are stained by anti-NGF antibodies in the endometriotic interstitium [69]. NGF is upregulated by inflammatory cytokines, such as TNF- $\alpha$ and IL-1 $\beta$. This neurotrophin is involved in persistent inflammatory pain by activating $\mathrm{MC}$ degranulation as well as cytokine production [70]. MCP1, which has a high level in the PF of endometriosis patients, is combined with CCR2 and participates in the process of mononuclear cell infiltration at the site of inflammation [3] (Fig. 3).

\section{Innervation and endometriosis-associated pain}

Pain is the most common symptom in endometriosis patients. This pain may be the result of nociceptive, inflammatory or neuropathic, mechanisms in the form of dysmenorrhea, dyspareunia, or dyschezia [71]. The neurotropic and neuroprotective activity of cytokines suggest that inflammation is one of the major causes of endometriosisassociated pain. Furthermore, anti-inflammatory medication can reduce endometriosis-related pain [72]. The presence of nerves in endometriotic lesions has been confirmed. In humans, murine models and rat models, ectopic endometrium implants develop sympathetic, parasympathetic, and sensory nerve fibers $[6,8,10,73]$. The ANS, which consists of the sympathetic nervous system and parasympathetic nervous system, was first described in 1916 by John $\mathrm{N}$ Langley as an essential mechanism that maintains homeostasis in organisms [74]. The female reproductive system and intestinal tract are imbued with a

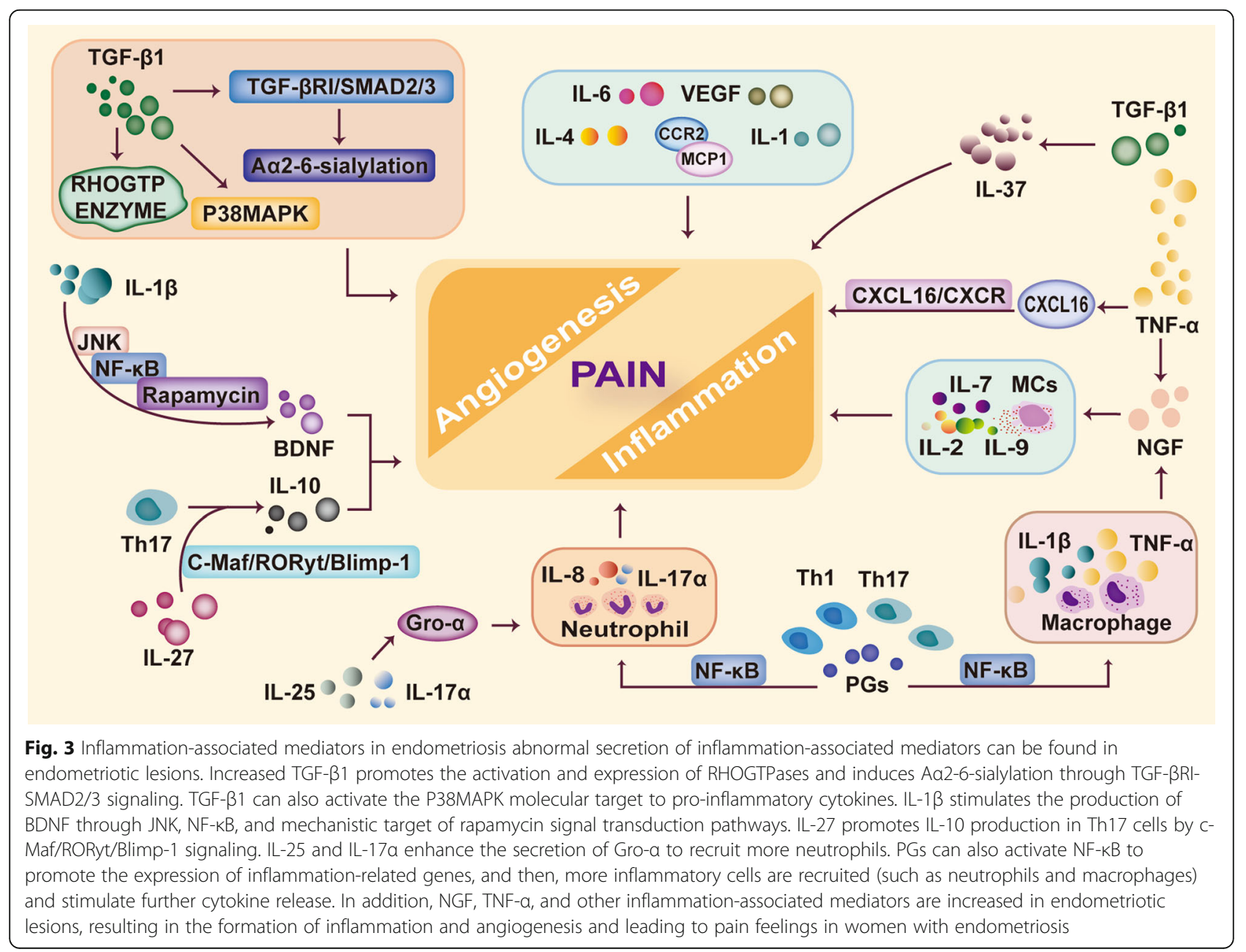


rich ground plexus of autonomic nerves. In addition to the regulation of vascular and nonvascular smooth muscle contractile activity, intestine movement, glandular secretions, and immune cell interactions, these plexuses can convey information to the central nervous system (CNS) regarding the internal environment and potential noxious stimuli $[75,76]$. Changes in the systemic levels of sex hormones (especially estrogen) can regulate the remodeling of uterine sympathetic nerves in nonpregnant females [75, 77]. Many studies have demonstrated that the imbalance among sympathetic, parasympathetic, and sensory innervation and the abnormal secretion of different cytokines can mediate neurogenesis and subsequent peripheral neuroinflammation in endometriosis [26].

\section{Peritoneal endometriosis}

Increased NFD can be detected in peritoneal endometriosis, not in the healthy peritoneum. These nerves mainly consist of A $\delta$ sensory, $C$ sensory, cholinergic, and adrenergic nerves. In particular, sensory nerve density is increased, while sympathetic nerve density is decreased [6, 9, 10]. In addition, Julia Arnold et al. [6] found that PF (collected from endometriosis patients) could induce an increased sprouting of sensory neuritis from dorsal root ganglia (DRG) and decreased neurite outgrowth from sympathetic ganglia (DRG and sympathetic ganglia were dissected from the spinal cord of the Valo specific pathogen-free eggs embryos). They hypothesized that the overexpression of NGF and IL-1 $\beta$ in PF could cause the overbalance of SP-positive nerve fibers, leading to a proinflammatory milieu that in turn affected PF in a vicious cycle [6]. Nerve fibers are located in or near endometriotic stromal cells and are colocalized with immature blood vessels. Furthermore, sympathetic nerve density is significantly higher in the adjacent tissue of lesions [78]. Infiltration of retroperitoneal endometriosis follows the pelvic pathways, while parasympathetic nerves are occasionally involved in infiltration. It is not clear why that happens because sympathetic nerves are present everywhere in the body, whereas endometriosis just occurs in pelvis preferentially rather than in all parts of the body [79].

The pelvic nerve emanates from the inferior hypogastric plexus and supplies sympathetic and parasympathetic innervation to the pelvic viscera. The inferior hypogastric plexus's efferent fibers reach to rectum, uterus, rectovaginal septa ventrally, and finally the deep vesicouterine ligament $[80,81]$. The vagus nerve provides parasympathetic nerve fibers to the entire abdomen proximal to a point approximated by the splenic flexure of the colon [82]. There is a complex environment in the pelvic and peritoneal cavity due to the anatomic structure and function of each visceral organ. Altered innervation in endometriosis lesions leads to changes in pelvic cavity innervation. Complex neural networks may transmit the pain produced by lesions to the brain.

\section{Ovarian endometriosis}

The human and mammalian ovaries are innervated by sympathetic, sensory, and a few parasympathetic nerves. Nerve fibers in ovarian endometriosis are a mixture of sensory, sympathetic, and parasympathetic nerve fibers. The ovary receives sympathetic innervation from the upper lumbar spinal segments via splanchnic nerve fibers and parasympathetic innervation via the vagus nerves. The autonomic axons reach the ovary through the ovarian nerve plexus and the superior ovarian nerve $[2,75,83]$. The study of Ricu M and colleagues showed that sympathetic nerves are involved in the control of early follicular growth [83]. The nerves of the fallopian tube basically regulate the smooth muscle contractility and ciliary beat activity of the fallopian tube [84]. The sympathetic innervation in fallopian tubes is regional variations. In the ampulla, nerves are known to be associated with blood vessels. The innervation of the isthmus is much denser and participates in neural control of the sphincter [75]. Parasympathetic nerves are relatively less dense in the fallopian tube and are mainly confined to the vasculature and muscular layer [75, 84].

Brett et al. [72] found a direct association between endometriosis-associated nerve fibers in peritoneal lesions and significantly higher menstrual pain. Ovarian endometriosis patients who reported the lowest pain were most unlikely to have endometriotic lesions with associated nerve fibers [72]. However, another study demonstrated multiple sympathetic and sensory nerves in ovarian endometriosis with a particularly high density (nerves $/ \mathrm{mm}^{2}$ ) of the sympathetic nerves [85]. Compared with women without endometriosis, PGP9.5 (a highly specific pan-neuronal marker), NPY (a marker of sympathetic nerve fibers), and VIP (a marker of parasympathetic nerve fibers) positive nerve fibers are decreased in the isthmus of the oviduct of endometriosis patients [84].

It can be assumed that when the ovarian innervation of endometriosis patients changes, ovarian electrophysiological activities as well as follicular activities are affected. In addition to the ovary, nerve fibers in the fallopian tube isthmus of endometriosis patients are decreased, leading to abnormal release of neurotransmitters. Subsequently, the smooth muscle contractility and ciliary beat activity of the fallopian tube are affected. Due to these various factors, normal female ovarian function is changed. The dysfunction of ovary and fallopian tube may lead to infertility in some women affected by endometriosis.

\section{Deep infiltrating endometriosis}

Hyperalgesia, a major characteristic of "neuropathic pain," and severe chronic pelvic pain are most commonly found 
in patients with deep infiltrating endometriosis (DIE) nodules $[8,86]$. DIE usually develops in anatomical sites with rich innervation. Typical sites are the rectovaginal septum, pararectal space, uterosacral ligament, rectum, ureters, diaphragm, and other less common sites [8, 87]. Sympathetic and sensory nerves are widely distributed in these areas under physiological conditions [11]. Some researchers have reported that immune-expression of NPY (sympathetic fibers) and VIP (parasympathetic fibers) are greatly higher in the uterosacral ligament and adjacent connective tissue in women with DIE. Transection of the uterosacral ligament to block these fibers can relieve pelvic pain $[2,88,89]$. There is no difference in NFD between lesions of the cul de sac, uterosacral ligament, and pelvic sidewall [8]. Other studies reported the loss of sympathetic nerve fibers in/near the lesions of intestinal endometriosis, especially in the mucosal and muscular layers. However, the density of sensory nerve fibers was unaltered in all of the analyzed areas near the endometriotic lesions $[2,11]$. NFD is highest in rectal lesions compared with that in other sites of DIE and six times higher than that in normal rectal walls. Pathological examination found that these nerve fibers usually invade ESCs. Polyclonal rabbit antihuman vesicular acetylcholine transporter (VAChT), which is a specific marker for cholinergic fibers, can be present in parasympathetic neurons. The VAChT enzyme is richly expressed in nerve fibers of rectal lesions but weak or absent in other endometriotic lesions [8]. Furthermore, GAP-43, a marker for neurite outgrowth, is selectively expressed in neurons associated with intestinal DIE lesions [3].

The enteric nervous system is vital for many gastrointestinal physiologic functions. The gut microbiota regulates the adult enteric nervous system via a major neuronal growth factor 5-hydroxytryptamine. 5-Hydroxytryptamine acts as a promoter of intestinal mucosa growth and also as a suppressor of inflammation in intestinal mucosa [90]. The human gut wall is richly innervated by sympathetic nerve fibers and by sensory fibers expressing the neuropeptide substance $\mathrm{P}$ (SP). In a mouse model, enteroendocrine cells (i.e., neuropod cells) synapse with vagal neurons to transduce gut luminal signals in milliseconds by using glutamate as a neurotransmitter to the brainstem [76]. Muscularis macrophages (MMs), which reside between the circular and longitudinal muscle layer of the bowel wall, are closely related to enteric neurons in the myenteric plexus. CSF-1 produced by enteric neurons modulates the inflammatory response of neighboring MMs [91].

As mentioned above, NFD in intestinal endometriosis is six times higher than that in the normal intestinal wall [8]. We can assume that neurogenesis is increased as endometriosis-related lesions develop. Due to the influence of various factors, the production of CSF-1 is enhanced after macrophage recruitment, contributing to the survival of macrophages [91]. MMs can regulate the adjacent inflammatory response [91]. Neuropod cells not only connect to the synapses of innate vagus nerve fibers but also connect to the synapses of new vagus nerve fibers and parasympathetic nerve fibers [76]. With the aggravation of endometriosis-associated inflammation, changes in the gut microbiota and inflammatory microenvironment [90], and the increased concentration of PG $[21,65]$, endometriosis-associated pain become more severe. These factors may contribute to greater pain. Next, the pain signal is transmitted to the brain through the interaction of neuropod cells and synapses [76]. At length, it may cause women with DIE (especially with intestinal endometriosis) to experience more pain sensation than women with other types of endometriosis.

\section{Neurotrophins and endometriosis-associated pain}

Endometriosis-associated pain is related not only to the number and distribution of nerves but also to some neurotrophins, especially BDNF and NGF.

In addition to the inflammation-associated changes in NGF mentioned above, the following changes of NGF are correlated with nerve fibers and neuropathic pain. NGF is a vital mediator of pain and inflammation [6]. NGF/TrkA signaling, implicated in neurodegenerative disorders (including Alzheimer's disease), chronic pain, inflammation, and cancer, plays a key role in neuronal development, growth, survival, and function [92]. NGF induces the expression of SP and calcitonin gene-related peptide, which are neuropeptides involved in modulation of central pain transmission [65]. Moreover, NGF promotes the sprouting of nociceptors, increases the number of sensory neurons, and contributes to persistent inflammatory pain [65]. In endometriosis, NGF expression is significantly higher in invasive lesions than in noninvasive lesions [22]. Inflammatory cells stained with an anti-NGF antibody in the endometriotic interstitium are commonly observed in human endometriosis and rat endometriosis models [69]. The immunointensity of NGF and its receptor TrkA is markedly elevated in the endometriotic epithelium and stroma of women with DIE. NGF immunointensity in the stroma is also significantly associated with local nerve bundle density and deep dyspareunia intensity $[3,93]$ Furthermore, suppression of NGF via siRNA in a surgically induced rat model of endometriosis inhibits both endometriotic lesion growth and NFD and reduces hyperalgesia [3]. These results suggest that abnormal innervation in endometriosis is associated with endometriosis-associated inflammation.

BDNF, another kind of neurotrophin, can promote cell growth, survival, and differentiation in several classes of neurons [68]. Moreover, nociceptor-derived BDNF, in addition to participation in the process of inflammation, 
has effects on promoting peripheral and CNS damaging pain [94]. BDNF is an important factor mediating the transition from acute to chronic pain [95]. The concentrations of BDNF in plasma and PF are significantly greater in women with endometriosis than in women without endometriosis. The expression levels of BDNF mRNA in ectopic lesions are markedly increased compared with those in eutopic and control endometrium. These results are correlated with endometriosis pain. IL$1 \beta$ can stimulate the production of BDNF via an IL-1 receptor-dependent fashion at the mRNA and protein levels. This effect of IL-1 $\beta$ is mediated by JNK, NF- $\mathrm{kB}$, and mechanistic target of rapamycin signal transduction pathways $[67,96]$. BDNF immunostaining is identified in adjacent sections, predominantly localized in the stromal and granular compartment of the DIE lesion, but not in the surrounding muscle tissue. In addition, immunohistochemistry revealed the in situ colocalization of macrophages, BDNF, and nerve fibers in endometriosis lesions [5]. These results suggest that BDNF plays a role in inflammatory pain and neuropathic pain through some signaling pathways. Moreover, when assessed for their ability to distinguish between women with revised Classification of the American Society of Reproductive Medicine stage 1 and 2 or 3 and 4 disease and controls, BDNF was the only presumptive marker capable of identifying stage 1 and 2 disease [97]. This finding suggests that plasma BDNF is a potentially useful clinical marker of endometriosis superior to CRP, CA-125, NT4/5 and NGF.

\section{ANS in other chronic inflammatory disorders}

The sympathetic nervous system (SNS) and parasympathetic nervous system (PNS) are classically balanced for maintaining homeostasis. It has been proven that ANS is disrupted in the process of various chronic inflammatory diseases (irritable bowel syndrome, IBD, RA, etc.), and the structure and function of the ANS are altered [10, 11, 98, 99].

The vagus nerve system is the major component of PNS, and it has a dual anti-inflammatory role through its afferent and efferent fibers [100]. SNS exhibits a more complex bidirectional influence. There are evidences that the loss of intestinal sympathetic innervation elicits an innate immunedriven inflammation [101]. In addition, the loss of sympathetic innervation is the hallmark of various acute and chronic inflammatory processes (such as colitis, arthritis, insulitis, etc.) in inflammatory lesions [102-104]. The SNS and vagus nerve have synergistic reaction via the splenic nerve to inhibit the release of TNF- $\alpha$ by macrophages of the peripheral tissues and the spleen [98]. As a result of the decreased concentration of sympathetic anti-inflammatory neurotransmitters, an inflammatory-promoting microenvironment is formed and triggers the following pain-generating signal pathway [99].
However, the anti-inflammatory role of SNS is controversial. The activation of the SNS can strengthen or suppress the activity of immune cells $[105,106]$. The reaction of immune cells to neurotransmitters is alterable depending upon the context of receptor engagement, such as microenvironment, cytokine milieu, activation state of cells, and expression pattern of neurotransmitter [106]. On the systemic level, the SNS has proinflammatory effects during the initial/early stage while anti-inflammatory effects in the later stages (e.g., AIDs and collagen-induced arthritis) $[6,10,106]$. Moreover, the imbalance of the ANS can be a predictor of various neuro-immune disorders. In particular, autonomic dysfunction, represented by low parasympathetic activity, precedes the development of some chronic inflammatory disorders, such as rheumatoid arthritis (RA). This finding suggests that autonomic dysfunction could be the etiopathogenesis of inflammatory disorders rather than a consequence of chronic inflammation [98].

There are many similarities between certain inflammatory disorders and endometriosis. Both IBD and endometriosis are chronic inflammatory disorders. The differential diagnosis between IBD and endometriosis is important in women with abdominal pain [107]. Simone Ferrero et al. demonstrated that sympathetic innervation of mucosa and the muscular layer around colorectal endometriotic lesions are significantly decreased [11]. These results are similar to those of CD [102], which suggests that similar changes in nerve fibers may be involved in the inflammatory response in $\mathrm{CD}$ and endometriosis [11]. Besides IBD, there are also many similarities between RA (a chronic inflammatory disorder) and endometriosis. The level of PGE2 is increased in synovial fluid of RA patients [108] and in PF of endometriosis women $[21,65]$. Similar to endometriotic PF and lesions, the concentration of M2 macrophages is increased in synovial tissues $[15,29,30,109]$. In addition, sympathetic nerve fibers are decreased and BDNF-positive cells are increased in RA synovium [110]. These findings suggest that RA may have something in common with endometriosis in pathogenesis. The following sections will briefly describe the mechanism of ANS changes in IBD and RA.

\section{Inflammatory bowel disease}

Inflammatory bowel disease (IBD), a chronic recurrent gastrointestinal tract disease, is divided into ulcerative colitis (UC) and Crohn's disease (CD) [100, 111]. The pathophysiology of IBD includes genetic, immunological, and environmental factors. IBD is characterized by production of various pro-inflammatory cytokines, such as TNF- $\alpha$ and IL-1 $\beta$, and ANS dysfunction [100, 112]. The intestine is dominated by the ANS, which is composed of the sympathetic and parasympathetic nervous 
systems. Sympathetic and parasympathetic nerve fibers provide afferent information from the gut to the brain and inform the CNS about the intestinal microenvironment $[113,114]$. It has been proven that emotional or psychological disorders and autonomic dysfunction can alter visceral sensitivity, usually by lowering the pain threshold $[115,116]$. The most common is stress, which can alter the brain-gut axis, leading to a wide range of gastrointestinal disorders, including IBD, irritable bowel syndrome, and other functional gastrointestinal diseases $[117,118]$ Moreover, stress acts as a vital factor in changing the ANS and can cause long-term modifications in sympathetic and vagus nerve balance [119]. Stress inhibits the vagus nerve and stimulates the SNS via autonomic-related projection neurons of the PVH to the dorsal motor nucleus of the vagus nerve and sympathetic preganglionic neurons of the spinal cord. Because of the anti-inflammatory properties of the vagus nerve through its afferent and efferent fibers, stress has proinflammatory properties [120].

The sympathetic dysfunction in CD and vagal dysfunction in UC have been reported [102, 113, 121]. The decreased sympathetic innervation in the intestinal wall of $\mathrm{CD}$ patients gives rise to the predominance of proinflammatory SP-positive nerve fibers compared with tyrosine hydroxylase $(\mathrm{TH})$-positive nerve endings [11, 102]. Sympathetic nerve fibers are increased in UC patients [113]. Other studies have also shown that the level of catecholamine (CA) increases in UC patients, while it decreases in CD patients [121].

The specific mechanism of the difference of changes of sympathetic nerves between $C D$ and UC is still not clear. However, more and more researchers agree that the SNS plays either pro- or anti-inflammatory role depending on the time point of SNS modulation [106, 122]. In the beginning of early inflammatory response, neurotransmitters (such as norepinephrine (NE)) of the SNS support chemotaxis and extravasation of leucocytes. At the same time, the concentration of NE can affect the metabolic state of macrophages. After leucocytes encountered in an inflammatory process, they begin to produce and secrete proinflammatory factors and sympathetic nerve repellent factors. Then release of neurotransmitters is inhibited and loss of sympathetic nerves occurs $[101,122]$. This may be the reason of alteration of sympathetic nerve fibers density in lesions of some inflammatory disorders in different periods.

It is generally believed that $\beta$-adrenergic receptor $(\beta$ AR) inhibits many immune cells of innate immune system (such as neutrophils, macrophages, etc.). Low concentration of NE can enhance TNF level of macrophage via $\alpha$-2-AR-mediated action or $\beta$-AR signaling [123]. High and low concentration of NE binds to $\beta$-AR and $\alpha$-AR respectively. In addition, NE can stimulate
Th2 immune responses by increasing IL-4, IL-6, and IL10 through $\beta$-AR signaling. On the contrary, Th1 immune responses (such as production of lymphocyte TNF, IL-2, and IFN- $\gamma$ ) are suppressed by $\beta$-AR signaling [122]. These results suggest that high NE might promote immune diseases with predominance of Th2 cytokines (such as UC), whereas low level of NE can activate innate immune system in chronic symptomatic phase (macrophage, neutrophils, etc.) of CD which is Th1 lymphocyte dominant [122]. The Th1 cells express high levels of $32-A R$, whereas expression is undetectable in Th2 cells [121]. Spleen is innervated by adrenergic pathways. The vagus nerve anti-inflammatory activity relies on ChAT-expressing $\mathrm{T}$ cells or B cells in the spleen and requires an intact splenic nerve (splenic nerve is one kind of sympathetic nerve) [124]. After activated of adrenergic splenic nerve, it can release NE into spleen activating memory $\mathrm{T}$ lymphocytes which produce acetylcholine (ACh) [125]. Stimulated release of ACh from T cells occurs following activation of $\beta 2$-AR by NE [126], and then, downstream responses are activated. UC can cause hyposplenism or compromise spleen function, placing these individuals at risk for developing "overwhelming post-splenectomy infections" [125]. Besides, the increased sympathetic nerve in UC lesions may also affect expression of $\beta 2$-AR. It may explain the mechanism of pathological sympathetic changes in UC. However, different environments of receptor engagement lead to altered responses of immune cells to neurotransmitters, and SNS can either enhance or inhibit the activity of cells associated with the acquired/adaptive immune system $[105,106]$. Consequently, it may explain the different role of the SNS in pathogenesis between $\mathrm{UC}$ and $\mathrm{CD}$.

In particular, $\mathrm{CD}$ has more similarities to DIE in terms of symptoms, changes in the number of nerves in the lesion, and other aspects. Both CD and endometriosis affect the bowel and can cause abdominal pain. Ileal endometriosis is a differential diagnosis of CD because both can cause inflammation, induration, thickening, and stricturing of the small bowel [127]. Sympathetic nerve fibers are decreased in all layers in CD patients [102]. Macrophages and fibroblasts in inflammatory lesions produce nerve repellent factors specific for sympathetic nerve fibers, such as Semaphorin 3C (Sema3C). Sema3C-positive crypts in the mucosa of $\mathrm{CD}$ are significantly increased and are negatively related to the density of mucosal sympathetic nerve fibers [102]. In addition, the reduction in parasympathetic tone can be found [115]. These nerve changes are similar to the DIE mentioned above $[2,11]$.

The loss of sympathetic nerves results in a decrease in the concentration of anti-inflammatory neurotransmitters. The neuropeptide SP plays a role in pro-inflammatory effects by inducing the secretion of pro-inflammatory 
cytokines, such as TNF, IL-6, and IL-8 [10, 102]. The vagal reflex pathway (the cholinergic anti-inflammatory pathway) has prominent effects on the regulation of the inflammatory response. The vagus nerves can perceive signals from the microbiota and then bring this intestinal information to the CNS, where they can integrate the information to produce the adaptive or inappropriate response. The latter can maintain the pathological condition of the digestive tract or be propitious to the development of disorders. The cholinergic anti-inflammatory pathway is mediated by synapses on enteric neurons that release ACh at the synaptic junction with macrophages through vagal efferent fibers. ACh binds to the $\alpha-7$-nicotinic ACh receptors of macrophages to inhibit the release in TNF. Accordingly, the decrease of parasympathetic tone in $\mathrm{CD}$ patients reduces the stimulation $\alpha-7$-nicotinic ACh receptors, which leads to an increase in cytokines such as TNF and consequently to persistent intestinal inflammation [115, 128]. The histochemistry results of neural tissue of the excised end of ileum have shown that transient receptor potential cation channel subfamily $\mathrm{V}$ member 1 (TRPV1) is increased. Multivariate regression analysis suggests that TRPV1immunorective fibers and MCs are correlated with abdominal pain score, proposing that these fibers might lead to visceral hypersensitivity [129]. The accumulation of $\mathrm{CD} 4^{+}$ $\mathrm{T}$ cells, induced by chronic inflammation in the mouse colon, and endogenous opioids has an effect on analgesia. This effect at least in part works through modulation of voltage-gated ion channels underlying action potential electrogenesis and TRPV1 [129, 130] (Fig. 4).

\section{Rheumatoid arthritis}

Rheumatoid arthritis (RA) has many common features with endometriosis, such as inflammation and autonomic

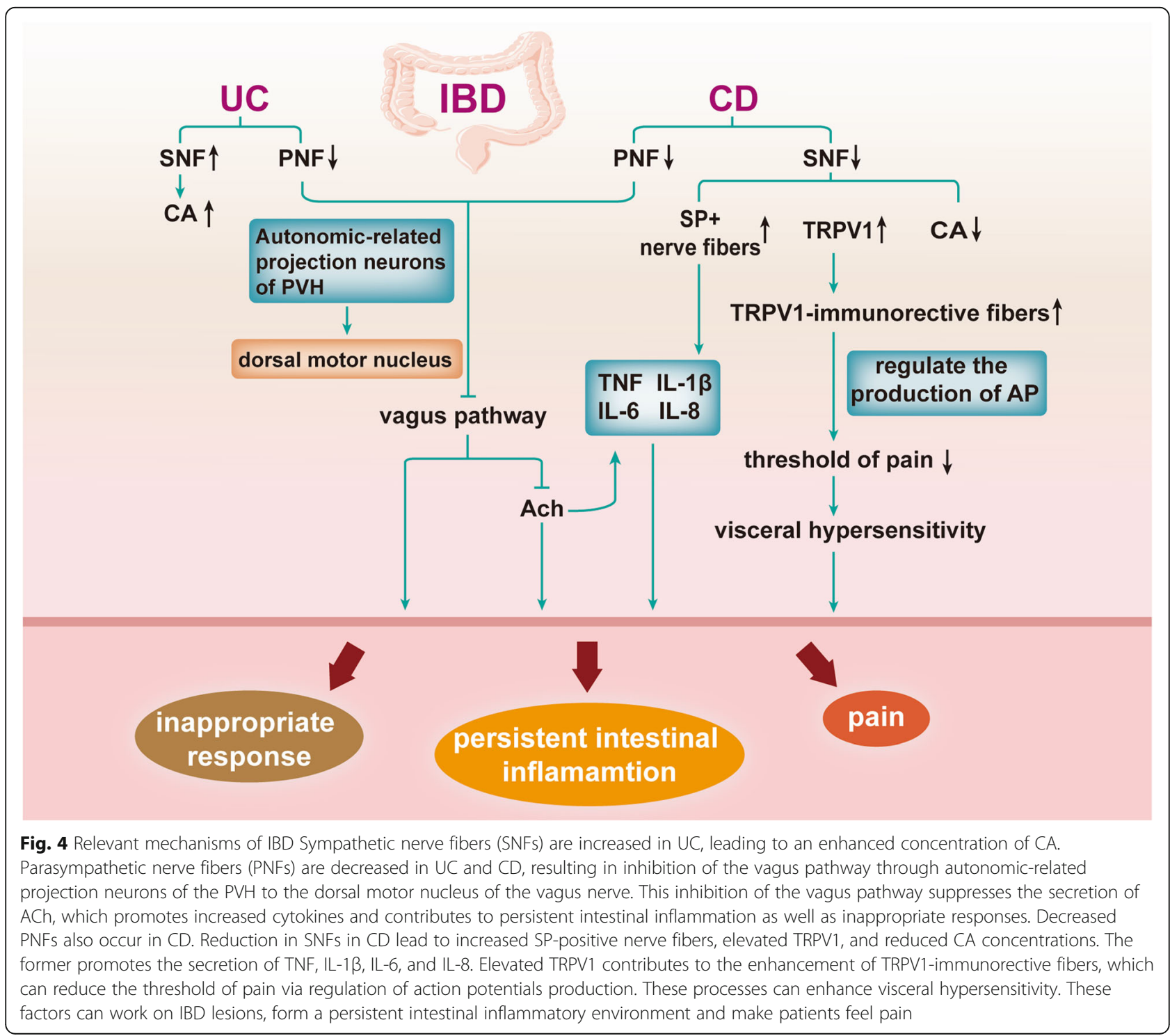


dysfunction. RA is a chronic inflammatory disease. The concentrations of macrophages and cytokines (such as TNF) are increased [109, 131]. Nerve repellent factors of sympathetic nerve fibers Sema3C and Sema3F can be found in the lesions of RA [103]. In addition to inflammation, autonomic dysfunction exists. Autonomic dysfunction occurs before the development of RA, and there is a causal relationship between autonomic nervous dysfunction and the development and progression of RA. This finding could be relevant for endometriosis and other immune-mediated inflammatory diseases. In the RA patients, the activity of PNS is reduced, whereas the SNS is overactive. In RA synovium, researchers can find a low density of sympathetic nerve fibers. Moreover, the level of NE is lower in RA patients than in arthritis patients [110, 132-134]. The depletion of CA before the onset of arthritis in an RA animal model plays an anti-inflammatory role, suggesting that $C A$ might promote the immune response before the onset of disease. The lower level of NE could lead to the activation of $\alpha$-AR types. Activation of $\alpha$-AR may lead to a more pro-inflammatory status, but little information is available $[132,134]$. In addition to the activation of $\alpha$ AR, $\beta 2$-AR is reduced in the mouse model of RA- collagen-induced arthritis (CIA) [135]. It has been proven that sympathetic neurotransmitter NE inhibits the inflammation of CIA by suppressing the differentiation and function of Th17 cells through $\beta 2-\mathrm{AR}$ signaling. Th17 cells can produce specific cytokines (such as IL17 and IL-22), leading to pro-inflammatory reactions. $\beta 2-\mathrm{AR}$, produced by $\mathrm{CD} 4^{+} \mathrm{T}$ cells, is downregulated in CIA. NE activation of $\beta 2$-AR reduces the transition from CIA-induced $\mathrm{CD}^{+}{ }^{+} \mathrm{T}$ cells to the Th17 phenotype. This activated $\beta 2$-AR-cAMP-PKA signaling in Th17 cells undermines the CIA-induced cell inflammatory response to exert the anti-inflammatory effect [135]. Furthermore, when the concentration of NE gets higher, NE binds to $\beta 2-\mathrm{AR}$ and transmits the information through GalphaS (Gos)-coupled proteins. However, this anti-inflammatory pathway can be disrupted in an inflammatory environment. The Gos-pathway (high cyclic AMP) of receptor coupling can switch to the Galphai (Gai)-pathway (low cyclic AMP) in RA mixed synovial cells, which is a pro-inflammatory signal [103].

The receptors of Sema3C and Sema3F are present on sympathetic nerve endings in the periphery. Their binding to their receptors leads to sympathetic nerve fiber repulsion in the neurite outgrowth assay [103]. TNF is an important inflammatory mediator. A study of Parkinson's disease demonstrated that overexpressed TNF can reduce the expression of tyrosine hydroxylase (TH) and that TNF is toxic to catecholaminergic neurons. Moreover, TNF inhibitors can downregulate various cytokines in a murine collagen type II-induced arthritis model [131].
Hence, a high level of TNF and the existence of nerve repellent factors of sympathetic nerve fibers might be the causes of the loss of sympathetic nerve and low concentration of NE (Fig. 5).

\section{Possible relationship between the ANS and endometriosis-associated inflammation}

As mentioned above, endometriosis has much in common with RA and IBD-chronic inflammation conditions, changes in inflammatory mediators, and the distribution of nerve fibers. The latter has been demonstrated in previous studies. However, it is not clear how the aberrant sympathetic and parasympathetic innervation in endometriosis is involved in the inflammatory reaction. Only a few studies support this phenomenon. In RA and IBD (especially in $C D$ ), relative mechanisms have been confirmed. According to these studies, we propose the possible relationship between the ANS and endometriosis-associated inflammation (Fig. 6).

\section{How does inflammation cause abnormal distribution and function of the ANS?}

Multiple inflammatory cells (such as macrophages, neutrophils, and MCs) are recruited to the lesion after the initiation of endometriosis, due to the inflammatory processes activated by the endometrial debris [24]. After that, neutrophils and MCs, various cytokines (TNF- $\alpha$, TGF- $\beta$, IL-1 $\beta$, IL-6, IL-8, IL-37, etc.), chemokines (MCP1, CXCL8, CCL2, CCL5, etc.), and other inflammatory mediators (BDNF, NGF, etc.) are secreted [5, 7, 8, 10, 14-17].

Among these cytokines, IL-1 $\beta$ promotes the production of BDNF through an IL-1 receptor-dependent fashion, mediated by JNK, NF- $\mathrm{KB}$, and the rapamycin signal transduction pathway $[5,67,96]$. The increased BDNF colocalizes with macrophages and nerve fibers, and can promote the growth, survival, and differentiation of several types of neurons [5, 68]. These factors interact with each other and participate in the development of inflammation as well as the formation of damaging pain in the peripheral and central nervous systems [94].

NGF is secreted by inflammatory cells and upregulated by cytokines (such as TNF- $\alpha$ and IL-1 $\beta$ ) $[5,7,10,70]$. The expression of NGF and its receptor TrkA is significantly enhanced in endometriotic epithelium and stroma $[3,22,93]$. NGF-TrkA signaling is involved in chronic pain and neuroinflammation [92]. NGF gives rise to the expression of SP and calcitonin gene-related peptide, contributing to the sprouting of nociceptors and increased number of sensory neurons $[6,65]$. Under the influences of BDNF and NGF mediated by cytokines, total NFD is elevated. Finally, increased NGF and BDNF lead to persistent inflammatory pain. 


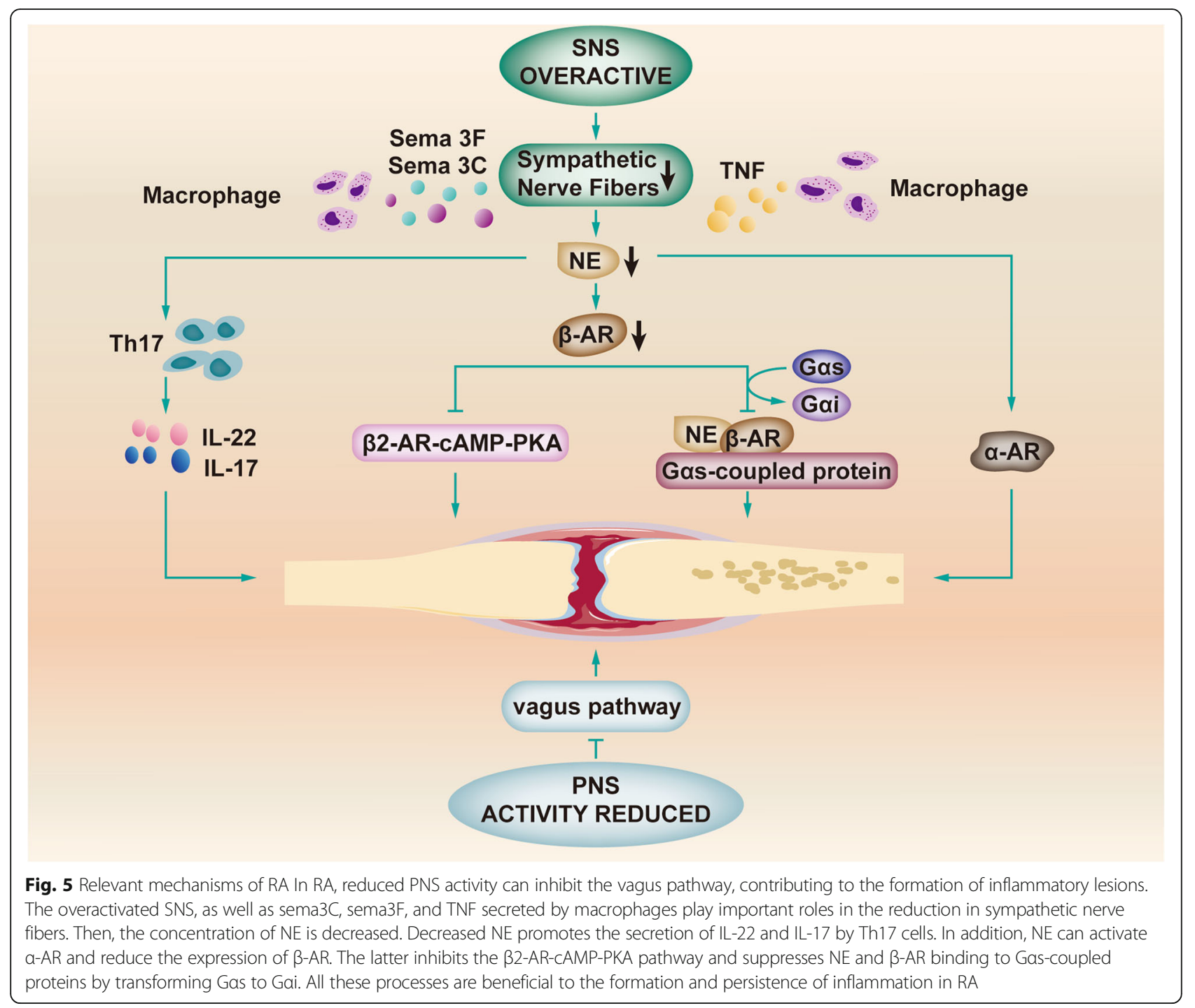

Macrophages produce nerve repellent factors (Sema3C and Sema3F, etc.) specific for sympathetic nerve fibers $[2,11]$. In addition, sympathetic nerve endings in the periphery have the receptors of Sema3C and Sema3F [103]. The binding to their specific receptors leads to sympathetic nerve fiber repulsion in the neurite outgrowth assay. Increased TNF- $\alpha$ secreted from inflammatory cells affects the early stage of endometriosis via activating local or systemic inflammation, and also has toxic effects on CA neurons [131]. Overexpression of Sema3C, Sema3F, and TNF will induce the loss of sympathetic nerve fibers in endometriosis.

In addition, we propose that abnormal secretions of cytokines, chemokines, and other mediators by the dysfunction of macrophage and MCs form a vicious cycle in endometriosis lesions. This cycle may aggravate the inflammatory neuropathic pain in endometriosis.
How does dysfunction of the ANS cause inflammation? The loss of sympathetic nerve fibers is a marker of the transition from acute to chronic inflammation [102-104]. This process leads to a reduction in the concentration of $\mathrm{CA}$, while the concentration of SP is elevated. Proinflammatory peptide SP can induce increased secretion of pro-inflammatory cytokines (such as TNF, IL-6, and IL8) $[10,11,65,102]$. In addition, CA sometimes can act as an anti-inflammatory neurotransmitter. Therefore, the reduction of CA and increase of SP can weaken the antiinflammatory effect and enhance the pro-inflammatory effect of endometriotic lesions, thus maintaining the inflammatory microenvironment as well as sustaining the development of inflammatory lesions.

The reduction of CA (the main component of reduction is NE) activates $\alpha$-AR while inhibits $\beta 2$-AR [132, 134, 135]. Normally, NE binds to the $\beta 2-A R$ and then transmits information via Gas-coupled proteins 


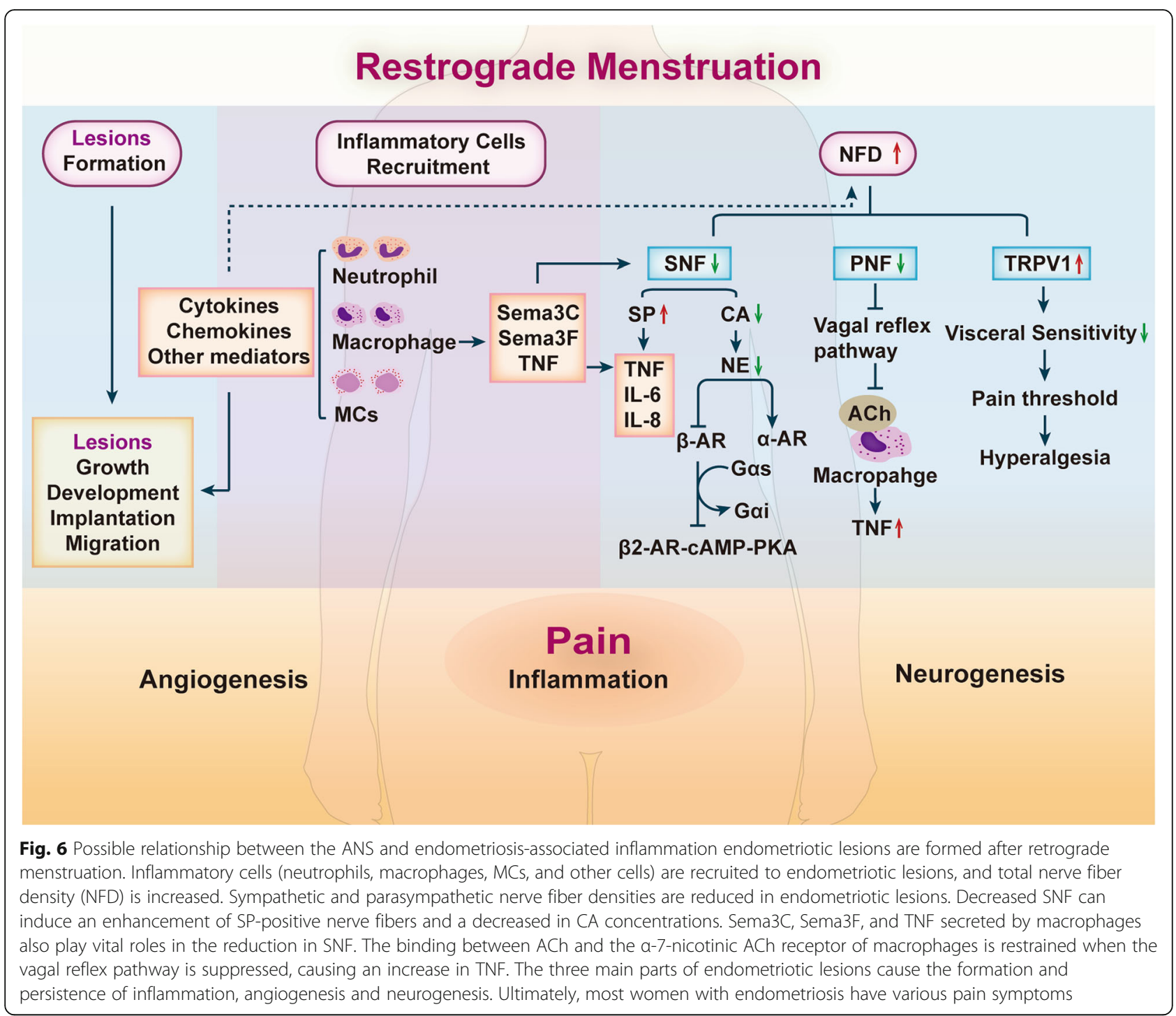

[103]. However, the inflammatory environment can disrupt this anti-inflammatory pathway. At the same time, the Gos-pathway (high cyclic AMP) of receptor coupling can switch to the Goi-pathway (low cyclic AMP), which is a pro-inflammatory signal [103]. Therefore, the low concentration of NE and downregulation of $\beta 2-\mathrm{AR}$ possibly lead to a pro-inflammatory response in lesions. It can be assumed that the reduced concentration of NE will lead to an enhancement of inflammation in endometriotic lesions through the aforementioned mechanisms.

However, CA does not always act as an anti-inflammatory neurotransmitter. It can also act as proinflammatory or in a dose/time-dependent biphasic manner. Depressed catecholO-methyltransferase (an enzyme that metabolizes catecholamines) can increase the levels of $\mathrm{NE}$ and epinephrine, which activate $\beta 2 / 3$ ARs to produce heightened pain sensitivity [136]. Instead, NE can activate $\beta$-AR to reduce LPSinduced inflammatory responses in bone marrow-derived macrophages. After sympathectomy, mRNA expression levels of pro-inflammatory cytokines are increased and mRNA expression level of anti-inflammatory cytokine IL-10 is decreased, suggesting that NE can play an antiinflammatory role in this situation [101]. Härle P et al. have demonstrated that the SNS has time-dependent opposing effects on the severity of CIA. The SNS has pro-inflammatory effects in early stage and confers anti-inflammatory effects in the later stage [137]. The bimodal effect of the SNS is dependent on the time point of immune system activation and the respective compartment [137].

The reduction in parasympathetic NFD and inhibition of the vagal reflex pathway alter the brain-gut axis $[117,118]$. After the transmission of changes in the intestinal microenvironment from gut to $\mathrm{CNS}$, an inappropriate response occurs. This response helps to maintain the pathological state of the intestine and promotes the development of 
disease. In addition, the concentration of $\mathrm{ACh}$, released by synapses of enteric neurons, is decreased. As a result, the binding between $\mathrm{ACh}$ and the $\alpha-7$-nicotinic ACh receptor of macrophages is restrained. This process leads to increased expression of cytokines such as TNF, maintaining persistent intestinal inflammation $[115,128]$. In addition, inflammatory mediators can stimulate TRPV1 and other peripheral nociceptors [129]. TRPV1-positive nerves induce the secretion of neurotransmitters from abnormal nerve fibers of endometriotic lesion. This process can in turn aggravate the local neurogenic inflammatory response.

The above changes in ANS can inhibit the antiinflammatory pathway and enhance the effect of the pro-inflammatory effect. Afterwards, the production of pro-inflammatory mediators is elevated, leading to inappropriate responses. These factors maintain the inflammatory pathological state of endometriotic lesions.

\section{How does the interaction between inflammation and the ANS participates in endometriosis?}

The possible relationship between inflammation and ANS has been mentioned above. In brief, inflammatory cells promote the production of cytokines, chemokines, and other noninflammatory factors. Elevated levels of neurotrophins lead to an increase in total NFD. Moreover, the sympathetic nerve repellent factors Sema3C and Sema3F also secreted, leading to sympathetic nerve fiber repulsion. As a result, the expression of SP is increased. These factors in turn promote the release of inflammatory mediators. Moreover, enhanced activation of NF- $\mathrm{KB}$ contributes to the elevated expression of inflammatory factors. This interaction of inflammation and the ANS promotes the development of inflammation in endometriotic lesions and has a significant impact on the occurrence and development of lesions and pain.

Thus, how does this relationship participate in the development of endometriosis? Additionally, how does this interaction cause pain and participate in the development of pain?

Angiogenesis and cell proliferation are vital to the development of endometriotic lesions. The immunepredominant phase and hormone-predominant phase contribute to the development and maintenance of endometriosis. Angiogenesis belongs to the initiation phase $(<72 \mathrm{~h}$ after disease initiation). After implantation of ectopic endometriotic lesions, a new vascular system is needed to maintain the survival and sustained growth of ectopic lesions. Angiogenesis is beneficial to the survival, growth, implantation, and migration of endometriotic lesions [17, 23, 24, 31, 32, 56-58]. In cancer, activated macrophages can increase the migration and invasion of tumor cells as well as anti-tumor immunity $[24,26]$. Analogously, in endometriosis, the recruitment of macrophages into endometriotic lesions leads to the production of PGE2/TGF- $\beta$, resulting in the inhibition of the immune response [3,10,20,22]. Macrophages can also co-occur with the recruitment of circulating endothelial progenitors and sustain their survival as well as the integrity of the vessel wall [24]. The hypoxic environment of local endometriotic lesions stimulates overexpression of COX-2, resulting in aberrant production of PGE2. PGE2 stimulates steroidogenesis, angiogenesis, and immunologic suppression in endometriosis. Moreover, hypoxic conditions promote angiogenesis through IL-8 from macrophages, contributing to the elevated migration of ESCs [4-6, 58]. Increased macrophages and neutrophils enhance the expression of VEGF and promote angiogenesis $[42,44]$. In addition, the development and distribution of vessels and nerve fibers are synchronous in the rat model of endometriosis and they are always concomitant [3]. Abundant angiogenesis also provides conditions for the growth of nerve endings. Both the vessels and nerves affect the development of endometriotic lesions. The abundant blood supply of neovascularization provides adequate nutrition for the growth of lesions and nerve endings, contributing to adhesion, invasion, and aberrant innervation of endometriosis.

Multiple inflammatory and noninflammatory mediators are essential for the development of endometriotic lesions. IL-10 contributes to proliferation and implantation of ectopic lesions. IL-8 is associated with cell proliferation and growth of endometrial cells and has chemotactic effects on neutrophils, resulting in the transformation of acute inflammation to chronic inflammation [17, 56-58]. The continuous activation of MCs and the production of IL- 4 and IL- 6 are related to the production and maintenance of persistent inflammation, leading to deterioration of endometriosis [19, 61]. NGF is involved not only in the growth of neonatal nerve fibers but also in the activation of MC degranulation. The occurrence and maintenance of persistent inflammation are related to sustained activation of MCs and the production of IL-4 and IL- $6[3,5,7,10]$. As a result, these effects contribute to the exacerbation of endometriosis. In addition to the involvement of neurogenesis, NGF can activate MC degranulation to produce more inflammation-associated mediators, such as PG [70]. PG activates NF- $\mathrm{kB}$ to promote the expression of inflammation-related genes [67]. Then chemokines are increased, and macrophages and neutrophils are recruited in endometriotic lesions, leading to the enhancement of chronic inflammation. RHOGTPases (related to cell migration and adhesion) activate P38MAPK through TGF- $\beta$, resulting in increased angiogenesis, development of endometriotic lesions, and aggravation of inflammation $[63,64]$. In addition, TNF- $\alpha$ has a toxic effect on the sympathetic nerve [131], leading to decreased secretion of anti-inflammatory mediators and increased production of 
pro-inflammatory mediators. All of these factors contribute to endometriosis progression and deterioration. The recruitment of neutrophils causes increased secretion of IL-17, leading to the proliferation of ESCs $[16,47,60]$. This process promotes cell adhesion. Cell adhesion impels the enhanced expression of TNF- $\beta 1$, IL- 1 , and TNF- $\alpha$ via the P38/ERK1/2 signaling pathway. This process contributes to the expression of IL-8 and VEGF [58]. These effects in turn promote the proliferation of ESCs and adhesion of vascular cells.

Changes of microenvironment and ANS in endometriosis participate in the mechanism of endometriosisassociated pain. Many aspects of inflammatory microenvironment can induce pain. Extensive activation of MCs causes more secretion of NGF, resulting in increased growth of nerve fibers. Next, nociceptors are activated, and peripheral nociceptive endings are sensitized. Ectopic implantation of the endometrium can release molecular signals that act on peripheral nociceptive endings, depolarizing them and generating AP. These effects can reduce the pain threshold so that harmless stimuli can activate high-threshold nociceptors. Moreover, NGF promotes sprouting of nociceptors and increases the number of sensory neurons, contributing to persistent chronic inflammatory pain. In addition, activated MCs release histamine, interleukin, TNF- $\alpha$, and PGs, causing neuropathic pain. The inflammatory factor IL- $1 \beta$ also reduces the mechanical nociceptor threshold and activates the nociceptive nerve. BDNF not only participates in endometriosis-associated inflammation but also promotes peripheral and central nervous systemic pain. BDNF plays a role in the relationship between inflammatory pain and neuropathic pain via some signaling pathway. During the inflammatory state, nerves carry damage signals to the brain, then the sensitized CNS release peptides into the local environment. Once $\mathrm{C}$ sensory nerve fibers transmitting the pain signal are activated, even if inflammation has been eliminated, continuous electrical activity and pain still exist. Increased TRPV1 in endometriotic lesions can regulate the generation of AP and induce persistent AP, leading to a change in visceral sensitivity and reduction in the threshold of pain. TRPV1-positive nerves trigger the release of neurotransmitters from peripheral tissues and enhance local neurogenic inflammation. Both promote the development of inflammation, produce hyperalgesia, and eventually produce endometriosis-associated pain.

\section{The influence of sex hormone on inflammation and ANS contribute to endometriosis}

It is known that endometriosis is an estrogen-dependent disease [1]. Compared with healthy women, local levels of estrogen are higher in menstrual blood and PF of patients with endometriosis [138]. As mentioned above, the imbalance of immune cells and abnormal innervation of nerve fibers (sympathetic, parasympathetic, and sensory nerve fibers) are proved in endometriotic lesions. So, whether estrogen is involved in endometriosisassociated inflammation and ANS changes?

Estrogen can regulate the recruitment of immune cells via $E R \alpha$ and $E R \beta$ in different ways, contributing to an abnormal inflammatory environment in endometriosis. Erin Greaves et al. found that all endometriotic lesionresident macrophages (in human tissues and mouse model of endometriosis) expressed ER $\beta$ and up to 20\% macrophages expressed ER $\alpha$ [139]. Another in vitro experiment has demonstrated that ER $\beta$ can elevate CCL2 through NF- $\mathrm{KB}$ in ESCs, and increased production of CCL2 leads to ER $\beta$-mediated macrophage infiltration [140]. Furthermore, we also reported that increased estrogen could recruit MCs and macrophages to endometriotic lesions in many ways [78].

Besides inflammation, there are evidences that estrogen also affects the change of nerve fibers. In murine uterus, estrogen can inhibit sympathetic neurite outgrowth through regulating BDNF synthesis [141]. Acute administration of $17 \beta$-E2 to adult ovariectomy rates lead to an $85 \%$ reduction in the density of myometrial sympathetic nerves at $24 \mathrm{~h}$ [75]. In addition, other studies have demonstrated that sympathetic nerves are reduced in myometrium of women with estrogen-dependent disease adenomyosis [142]. The sympathetic denervation of rat myometrium is correlated with estrogen-induced collagen reorientation [143]. Longterm exposure to estradiol-17 $\beta$ leads to decreased cholinergic innervation in pig ovary [144]. Cyclic variations of sex hormone strongly influence uterine innervation, especially sympathetic nerves. The level of NA is also affected by this fluctuation [75]. In murine uterus, E2 regulates the synthesis of BDNF (exert a repulsive effect on sympathetic neurites through $\mathrm{p} 75^{\mathrm{NTR}}$ ) to influence neurogenic properties. BDNF activates sphingomyelinase and increases intracellular ceramides level through $\mathrm{p} 75^{\mathrm{NTR}}$ receptors presented on sympathetic axons [75]. This process in turn impedes sympathetic outgrowth [75]. NGF also plays a role in the change of nerve fibers in endometriosis. It is essential for the development and survival of adrenergic and sensory neurons both in CNS and peripheral nervous systems. TrkA and $\mathrm{p} 75^{\mathrm{NTR}}$ are required for carrying out the modulatory effects of sympathetic neurons of NGF. Interestingly, the survival of sympathetic neurons loss dependence on NGF, but axonal growth of sympathetic neurons remains under the influence of NGF. In high concentration of E2 environment, increased NGF prefers to promote axonal growth of sensory nerve fibers, and inhibit mature sympathetic ganglia outgrowth $[75,78]$. As a consequence, estrogen is important in the regulation of abnormal innervation in female reproductive system. 
According to the evidence discussed above, we proposed that estrogen can influence the interaction of inflammation and nerve fibers in endometriosis. In our previous study [145], we have reported that increased E2 could activate MCs to secrete NGF leading to sensitize dorsal root ganglion cells. After peripheral axon injury, neuron releases exosomal miR-21-5p mediated by estrogen signaling pathway. After that, macrophage phagocytizes miR-21-5p, which contributes to the infiltration of macrophages toward peripheral nerves. At the same time, peripheral nerves also secrete CSF-1 and CCL2 to enhance macrophages migration to endometriotic lesions [145]. In an animal experiment, rat DRG, treated with E2, can increase mRNA level of CCL2, which stimulates the migration of CSF-1-differentiated macrophages. In turn, macrophages treated with E2 can elevate concentrations of BDNF and NT3, promoting neurite outgrowth from ganglia explants [139]. According to these evidences, we suggest that estrogen not only influences the number and function of immune cells but also mediates the change of ANS in endometriotic lesions. More importantly, estrogen can influence the interaction of inflammation and nerve fibers in endometriosis.

As mentioned above, inflammation, or changes of ANS and sensory nerve fibers, can cause the development of endometriotic lesions and promote endometriosisassociated pain. According to statistics, $60 \%$ of women with painful periods suffer from endometriosis. Dysmenorrhea, pelvic pain, dyspareunia, and dyschezia are the most common pain symptoms of endometriosis, which seriously affect women's quality of life, career, and daily activities [146, 147]. In order to improve the quality of life of women with endometriosis, several drugs are commonly used in clinic to relieve pain and inhibit the development of lesions.

First-line and second-line medicine currently available for endometriosis-associated pain include nonsteroidal anti-inflammatory drugs (NSAIDs), hormone therapy, and gonadotropin-releasing hormone $(\mathrm{GnRH})$ agonists and antagonists [148]. NSAIDs are the most commonly used first-line medicine. It works by blocking the COX enzyme, which is important for the production of inflammatory factors [146]. However, if used for a long time, the risk of hypoestrogenic adverse effects, negative calcium balance, and osteoporosis increases [149].

$\mathrm{GnRH}$ antagonist is a type of prevalent medicine for endometriosis. GnRH antagonist downregulates the release of follicle stimulating hormone and luteinizing hormone via blocking the GnRH receptor in pituitary cells, leading to the suppression of ovulation [145]. GnRH agonists and antagonists are effective in the relief of endometriosisassociated pain, but they are also associated with tolerable hypoestrogenic adverse effects (vasomotor symptoms, genital hypotrophy, and mood instability) and negative calcium balance with an increased risk of osteopenia [149, 150]. Hormonal drugs can relieve pain in more than $90 \%$ of endometriosis patients [151]. These drugs inhibit ovulation and menstruation and have similar beneficial effects against pain. However, only estrogen-progestins and progestins have safety, tolerability, and cost profiles that allow longterm use [150].

Dienogest (DNG), to be marketed in mainland China, is a steroidal fourth-generation selective progestin that combines the pharmacologic properties of 19-nortestosterone, a derivative of progesterone. DNG combines potent progestogenic efficacy and moderate estrogen-suppressive effects to effectively reduce the growth of endometrial-like tissue, combined with anti-inflammatory, antiproliferative, and antiangiogenic effects. This treatment modulates the production and metabolism of PGs in a way that is antiinflammatory. Moreover, the use of DNG is associated with proinflammatory cytokine and chemokine production, as well as growth factor biosynthesis and signal kinases, which are responsible for the control of inflammation. Although DNG produces other progesterone-like side effects (e.g., weight gain, increased blood pressure, breast tenderness, and nausea), it has no androgenic side effects and has little effect on metabolic and lipid parameters. As a result, DNG has been shown to be well tolerated [152, 153].

In addition to inflammatory mediators mentioned above, a laboratory-based study found that use of hormonal therapy is associated with reduced NFD in endometriosis (especially in DIE) [154]. Progesterone can increase vaginal sympathetic (but not parasympathetic) nerve terminals in female Sprague-Dawley rats, whereas E2 can reduce innervation in progesteronetreated rat and untreated rat [155]. These effects of progesterone in nerve fibers may also relieve pain in women with endometriosis who use progesterone.

\section{Potential implication of ANS and inflammation interaction in the management of endometriosis treatment}

The treatment objectives of this chronic inflammatory disease are to relieve endometriosis-associated chronic pain, and to achieve pregnancy successfully in infertile women [156]. There are many methods for treating endometriosis, including medical treatment and surgical treatment [146]. However, the current treatment methods are not as effective as expected. Although pain can be managed through pharmacological inhibition of ovulation and menstruation, medical therapy is symptomatic not cytoreductive. In addition, surgery is usually associated with pain relief, but its effect is always temporary [149].

The effects of surgery on pain are often temporarily satisfactory. Peritoneal implants can be safely coagulated or excised with similar benefits. According to the progress of endometriosis or requirements in ovarian endometriosis 
patients, conservative ovarian surgery or excision of ovarian endometriomas is selected. For DIE patients, excisional surgery is the best way for women with symptomatic DIE especially intestinal endometriosis. Good pain relief is generally reported during the first year after bowel resection for DIE [149, 157].

In addition to the main drugs for endometriosis mentioned in the previous section (part 5 "The Influence of Sex Hormone on Inflammation and ANS Contribute to Endometriosis"), there are other medicines that can relief pain through affecting inflammatory response. Raloxifene can decrease M1 monocytes, macrophage density, and the NF- $\mathrm{kB}$ response associated with a decrease in NO, IL-1 $\beta$, and IL- 6 production. This property can make raloxifene effective in the treatment of chronic pelvic pain for endometriotic patients [145]. Pesveratrol is a new type of natural phenolic drug for the prevention and treatment of endometriosis. The basic mechanism of effect is considered to be anti-inflammatory reactions. Pesveratrol can inhibit the synthesis of PGs through inhibiting COX enzyme synthesis and activating immune cells and pro-inflammatory cytokines [156].

In view of the adverse reactions of the above drugs, there is an urgent need for innovative and more effective treatment methods. Selective estrogen receptor modulators provide a novel treatment strategy for endometriosis, causing both agonistic and antagonistic activity at the ER [145]. Glucosaminyl muramyl dipeptide prevents hyperactivation of macrophages to weaken inflammation in lesions [26]. In addition, antagonizing the EP4 receptor signal not only inhibits the proliferation of endometrial cells induced by PGE2 but also regulates anti-inflammatory activity and alleviates inflammatory pain. Moreover, this receptor is present in sensory nociceptive nerve fibers. Pain can enhance the expression of the EP4 receptor in dorsal root ganglion neurons. Therefore, human PGE2 receptor subtype 4 antagonists may be an effective new drug in the treatment of endometriosis [157].

Considering the similar mechanism among IBD, RA, and endometriosis described in the above section, we hope to find some new and more effective methods for endometriosis by referring to the treatment methods of these disorders. Infliximab is a TNF- $\alpha$ inhibitor. The clinical effect of infliximab is good in RA, although it has high costs [158]. Applying infliximab to treatment of endometriosis may not only inhibit TNF to control inflammation but also reduce the toxic effect of TNF on sympathetic nerve fibers. However, a review of drug development of endometriosis has described that this drug failed midway during development [159]. Hyaluronic acid (HA) can bind to the cell surface adhesion molecule CD44. CD44 is overexpressed in activated macrophages and synoviocytes under RA conditions. The hyaluronic acid-methotrexate conjugate can release methotrexate in inflamed tissue, resulting in downregulation of inflammatory cytokine levels and a reduction in cartilage damage in arthritic mice [158]. The concentration of soluble CD44 in the serum and PF of endometriotic patients is higher than that of healthy women $[160,161]$. According to this mechanism of hyaluronic acid-methotrexate, is it possible to consider designing a drug with a similar structure for endometriosis? Peptidyl arginine deiminase 4 (PAD4), an initiator of citrullination, is an important therapeutic target for inflammatory diseases. In-depth studies have been carried out on tumors and multiple inflammatory disorders (such as RA). The expression of PAD4 is regulated by an ER $\alpha$-dependent mechanism. Estrogen can regulate the expression of PAD4 via classical and nonclassical pathways at the transcriptional level. Moreover, PAD2 is expressed in the female genital tract and regulated by estrogen and epidermal growth factor in reproductive tissues [162]. As a result, PADs may be a new therapeutic target for endometriosis.

In neuropathways, vagal nerve stimulation can reduce serum IL-6 concentrations significantly in RA. The level of reduction is related to the degree of RA improvement [163]. This treatment method may lessen concentrations of IL-6 and other cytokines, which can weaken inflammation in endometriotic lesions. In terms of immune homeostasis, great progress has been made in the study of dendritic cell (DC) therapeutics in RA mouse models. In CIA models, the use of low doses of semimature DCs can suppress disease progression by elevating the Treg population and inhibiting antigen-specific Th1- and Th7mediated immunity. Treatment of CIA mice with tolerogenic DCs modified by tacrolimus significantly inhibited the severity and progression of disease by altering the proportion of Th1 and Th17 cells in the spleen [163]. The development of endometriosis is dependent on the presence of endogenous DCs. Mannose receptors on peritoneal DCs can enhance the phagocytosis of dead ESCs. DCs secrete IL-1 $\beta$ and IL-6 promoting the escape of immune surveillance and Th17 differentiation. Moreover, markedly decreased DCs cause larger endometriosis-like lesions and significantly reduce the activation of T lymphocytes [164]. In brief, the methods of DC therapeutics may be used for reference in endometriosis treatment.

However, there is still an urgent need to identify new targets and to further develop new drugs for endometriosis treatment to control the development of endometriosis and improve the quality of life of endometriotic patients.

\section{Conclusion}

Endometriosis is a complex, estrogen-dependent, and chronic inflammatory gynecological disease, associated with infertility and endometriosis-associated pain [26, 99]. Although the mechanisms require clarification, according to 
current studies, many researchers believe that inflammation and the changes of nerves in endometriotic lesions are related to the occurrence and development of endometriosis. The interaction between inflammation and nerves can aggravate endometriosis and is closely correlated with endometriosis-associated pain. Inflammation is considered to be a significant cause of pain because of the neurotropic and neuroprotective activities of cytokines [72]. In addition, abnormal innervation leads to changes in neurotransmitters and aberrant secretion of inflammatory factors. These changes can mediate neurogenesis and subsequent peripheral neuroinflammation in endometriosis [26].

The rich ground plexus of autonomic nerves is widely distributed in the female reproductive system, regulating glandular secretion, immune cell interactions, etc. This plexus also conveys information to the CNS regarding the internal environment and potential noxious stimuli [75]. Changes in the systemic levels of estrogen can regulate the remodeling of the uterine sympathetic nerve in nonpregnant females and promote the secretion of chemokines from peripheral nerves $[75,76,145]$. Due to the alterations of sympathetic and parasympathetic innervation and function in endometriotic lesions, the effects of anti-inflammation of the sympathetic and vagal reflex pathways are restrained, contributing to the formation and development of the pro-inflammatory microenvironment. Although certain mechanisms of endometriosis are still unclear, we can draw on the experience of studies of IBD, RA, and other chronic inflammatory disorders to determine a breakthrough aspect for further endometriosis studies and to provide some clues for new therapeutic targets.

\section{Abbreviations}

Ach: Acetylcholine; AIDs: Inflammatory autoimmune diseases;

ANS: Autonomic nervous system; AR: Adrenergic receptor; BDNF: Brainderived neurotrophic factor; CA: Catecholamine; CD: Crohn's disease; CHUK: Conserved helix-loop-helix ubiquitous kinase; CIA: Collagen-induced arthritis; CNS: Central nervous system; DCs: Dendritic cells; DIE: Deep infiltrating endometriosis; DNG: Dienogest; DRG: Dorsal root ganglia; E2: Estradiol; ER: Estrogen receptor; ESCs: Endometrial stromal cells; GnRH: Gonadotropin-releasing hormone; Gai: Galphai; Gas: GalphaS; IBD: Inflammatory bowel disease; IFN: Interferon; IL: Interleukin; JNK: c-Jun Nterminal kinase; LPMs: Large peritoneal macrophages; MCs: Mast cells; MMs: Muscularis macrophages; NFD: Nerve fiber density; NFKBIA: NF-kB inhibitor alpha; NGF: Nerve growth factor; PAD: Peptidyl arginine deiminase; PF: Peritoneal fluid; PGs: Prostaglandins; PNFs: Parasympathetic nerve fibers; PNS: Parasympathetic nervous system; RA: Rheumatoid arthritis;

SNFs: Sympathetic nerve fibers; SNS: Sympathetic nervous system; SP: Neuropeptide substance P; SPMs: Small peritoneal macrophages; TGF$\beta$ : Transforming growth factor- $\beta$; TH: Tyrosine hydroxylase; TNF: Tumor necrosis factor; TRPV1: Transient receptor potential cation channel subfamily $\checkmark$ member 1; UC: Ulcerative colitis; VAChT: Vesicular acetylcholine transporter; VEGF: Vascular endothelial growth factor

\section{Acknowledgments}

Not applicable.

\section{Authors' contributions}

WY and LY contributed equally to this work. WY and LY were involved in the acquisition of the data, analysis of the data, and drafting of the manuscript.
LH and DY collated the data of IBD and RA. LY and YS were involved in the critical revision of the manuscript. All authors read and approved the final manuscript.

\section{Funding}

This work was supported by (1) Natural Science Foundation of Guangdong Province, China (Grant No. 2016A030310151), (2) Student Innovation Training Program of Sun Yat-Sen University (Grant No. 201901097), (3) Special Funds for the Cultivation of Guangdong College Students' Scientific and Technological Innovation ("Climbing Program" Special Funds, pdjh2019a0004), and (4) National Natural Science Foundation of China (Youth Science fund) (Grant No. 81701416).

\section{Availability of data and materials}

The data supporting the conclusion of this article is included within the "References" section.

Ethics approval and consent to participate

Not applicable.

\section{Consent for publication}

Not applicable.

\section{Competing interests}

The authors declare that they have no competing interests.

\section{Author details}

'Department of Obstetrics and Gynecology, First Affiliated Hospital of Sun Yat-Sen University, No. 58, the 2nd Zhongshan Road, Yuexiu District, Guangzhou 510080, Guangdong, China. ${ }^{2}$ Zhongshan School of Medicine, Sun Yat-Sen University, Guangzhou 510089, China.

Received: 25 June 2019 Accepted: 20 February 2020

Published online: 07 March 2020

\section{References}

1. Symons LK, Miller JE, Kay VR, Marks RM, Liblik K, Koti M, Tayade C. The Immunopathophysiology of endometriosis. Trends Mol Med. 2018;24:748-62.

2. Morotti M, Vincent K, Brawn J, Zondervan KT, Becker CM. Peripheral changes in endometriosis-associated pain. Hum Reprod Update. 2014;20:717-36.

3. McKinnon BD, Bertschi D, Bersinger NA, Mueller MD. Inflammation and nerve fiber interaction in endometriotic pain. Trends Endocrinol Metab. 2015;26:1-10.

4. Zhang T, De Carolis C, Man G, Wang CC. The link between immunity, autoimmunity and endometriosis: a literature update. Autoimmun Rev. 2018;17:945-55.

5. Yu J, Francisco A, Patel BG, Cline JM, Zou E, Berga SL, Taylor RN. IL-1 beta stimulates brain-derived neurotrophic factor production in eutopic endometriosis stromal cell cultures: a model for cytokine regulation of neuroangiogenesis. Am J Pathol. 2018;188:2281-92.

6. Arnold J, Barcena DAM, Ruster C, Vercellino GF, Chiantera V, Schneider A, Mechsner S. Imbalance between sympathetic and sensory innervation in peritoneal endometriosis. Brain Behav Immun. 2012;26:132-41.

7. Tai FW, Chang CY, Chiang JH, Lin WC, Wan L. Association of pelvic inflammatory disease with risk of endometriosis: a nationwide cohort study involving 141,460 individuals. J Clin Med. 2018;7(11):379.

8. Wang G, Tokushige N, Markham R, Fraser IS. Rich innervation of deep infiltrating endometriosis. Hum Reprod. 2009;24:827-34.

9. Mechsner S, Kaiser A, Kopf A, Gericke C, Ebert A, Bartley J. A pilot study to evaluate the clinical relevance of endometriosis-associated nerve fibers in peritoneal endometriotic lesions. Fertil Steril. 2009;92:1856-61.

10. Coxon L, Horne AW, Vincent K. Pathophysiology of endometriosisassociated pain: a review of pelvic and central nervous system mechanisms. Best Pract Res Clin Obstet Gynaecol. 2018;51:53-67.

11. Ferrero S, Haas S, Remorgida V, Camerini G, Fulcheri E, Ragni N, Straub RH, Capellino S. Loss of sympathetic nerve fibers in intestinal endometriosis. Fertil Steril. 2010;94:2817-9.

12. Greaves E, Cousins FL, Murray A, Esnal-Zufiaurre A, Fassbender A, Horne AW, Saunders PT. A novel mouse model of endometriosis mimics human phenotype and reveals insights into the inflammatory contribution of shed endometrium. Am J Pathol. 2014;184:1930-9. 
13. Halme J, Hammond MG, Hulka JF, Raj SG, Talbert LM. Retrograde menstruation in healthy women and in patients with endometriosis. Obstet Gynecol. 1984;64:151-4.

14. Tran LV, Tokushige N, Berbic M, Markham R, Fraser IS. Macrophages and nerve fibres in peritoneal endometriosis. Hum Reprod. 2009;24: 835-41.

15. Bacci M, Capobianco A, Monno A, Cottone L, Di Puppo F, Camisa B, Mariani M, Brignole C, Ponzoni M, Ferrari S, et al. Macrophages are alternatively activated in patients with endometriosis and required for growth and vascularization of lesions in a mouse model of disease. Am J Pathol. 2009; 175:547-56.

16. Takamura M, Osuga Y, Izumi G, Yoshino O, Koga K, Saito A, Hirata T, Hirota Y, Harada M, Hasegawa A, Taketani Y. Interleukin-17A is present in neutrophils in endometrioma and stimulates the secretion of growthregulated oncogene-alpha (Gro-alpha) from endometrioma stromal cells. Fertil Steril. 2012;98:1218-24.

17. Sikora J, Smycz-Kubanska M, Mielczarek-Palacz A, Kondera-Anasz Z. Abnormal peritoneal regulation of chemokine activation - the role of IL-8 in pathogenesis of endometriosis. Am J Reprod Immunol. 2017;77(4). https:// doi.org/10.1111/aji.12622.

18. Chang KK, Liu LB, Jin LP, Zhang B, Mei J, Li H, Wei CY, Zhou WJ, Zhu XY, Shao J, et al. IL-27 triggers IL-10 production in Th17 cells via a C-Maf/ RORgammat/Blimp-1 signal to promote the progression of endometriosis. Cell Death Dis. 2017;8:e2666.

19. Wang $F$, Wang $H$, Jin $D$, Zhang $Y$. Serum miR-17, IL-4, and IL-6 levels for diagnosis of endometriosis. Medicine (Baltimore). 2018;97:e10853.

20. Yao C, Narumiya S. Prostaglandin-cytokine crosstalk in chronic inflammation. Br J Pharmacol. 2019;176:337-54.

21. McAllister SL, Giourgas BK, Faircloth EK, Leishman E, Bradshaw HB, Gross ER. Prostaglandin levels, vaginal innervation, and cyst innervation as peripheral contributors to endometriosis-associated vaginal hyperalgesia in rodents. Mol Cell Endocrinol. 2016;437:120-9.

22. Orellana R, Garcia-Solares J, Donnez J, van Kerk O, Dolmans MM, Donnez O. Important role of collective cell migration and nerve fiber density in the development of deep nodular endometriosis. Fertil Steril. 2017;107:987-95.

23. Burns KA, Thomas SY, Hamilton KJ, Young SL, Cook DN, Korach KS. Early endometriosis in females is directed by immune-mediated estrogen receptor alpha and IL-6 cross-talk. Endocrinology. 2018;159:103-18.

24. Capobianco A, Rovere-Querini P. Endometriosis, a disease of the macrophage. Front Immunol. 2013:4:9.

25. Pepe G, Locati M, Della TS, Mornata F, Cignarella A, Maggi A, Vegeto E. The estrogen-macrophage interplay in the homeostasis of the female reproductive tract. Hum Reprod Update. 2018;24:652-72.

26. Wu J, Xie H, Yao S, Liang Y. Macrophage and nerve interaction in endometriosis. J Neuroinflammation. 2017;14:53.

27. Qian BZ, Pollard JW. Macrophage diversity enhances tumor progression and metastasis. Cell. 2010;141:39-51.

28. Yuan M, Li D, An M, Li Q, Zhang L, Wang G. Rediscovering peritoneal macrophages in a murine endometriosis model. Hum Reprod. 2017;32 94-102.

29. Shao J, Zhang B, Yu JJ, Wei CY, Zhou WJ, Chang KK, Yang HL, Jin LP, Zhu $X Y$, Li MQ. Macrophages promote the growth and invasion of endometrial stromal cells by downregulating IL-24 in endometriosis. Reproduction. 2016; 152:673-82.

30. Nie MF, Xie $\mathrm{Q}, \mathrm{Wu} Y \mathrm{YH}$, He H, Zou $L$, She $X L$, Wu XQ. Serum and ectopic endometrium from women with endometriosis modulate macrophage M1/ M2 polarization via the Smad2/Smad3 pathway. J Immunol Res. 2018;2018: 6285813.

31. Sekulovski N, Whorton AE, Shi M, MacLean Jl, Hayashi K. Endometriotic inflammatory microenvironment induced by macrophages can be targeted by niclosamidedagger. Biol Reprod. 2019;100:398-408.

32. Ponce $C$, Torres M, Galleguillos C, Sovino H, Boric MA, Fuentes A, Johnson MC. Nuclear factor kappaB pathway and interleukin-6 are affected in eutopic endometrium of women with endometriosis. Reproduction. 2009;137:727-37.

33. Binda MM, Donnez J, Dolmans MM. Targeting mast cells: a new way to treat endometriosis. Expert Opin Ther Targets. 2017;21:67-75.

34. Menzies FM, Shepherd MC, Nibbs RJ, Nelson SM. The role of mast cells and their mediators in reproduction, pregnancy and labour. Hum Reprod Update. 2011;17:383-96.

35. Olivera A, Beaven MA, Metcalfe DD. Mast cells signal their importance in health and disease. J Allergy Clin Immunol. 2018;142:381-93.
36. Bungum HF, Nygaard U, Vestergaard C, Martensen PM, Knudsen UB. Increased IL-25 levels in the peritoneal fluid of patients with endometriosis. J Reprod Immunol. 2016;114:6-9.

37. Gonzalez-de-Olano D, Alvarez-Twose I. Mast cells as key players in allergy and inflammation. J Investig Allergol Clin Immunol. 2018;28:365-78.

38. Mortaz E, Amani S, Mumby S, Adcock IM, Movassaghi M, Folkerts J, Garssen J, Folkerts G. Role of mast cells and type 2 innate lymphoid (ILC2) cells in lung transplantation. J Immunol Res. 2018;2018:2785971.

39. Wilcock A, Bahri R, Bulfone-Paus S, Arkwright PD. Mast cell disorders: from infancy to maturity. Allergy. 2019;74:53-63.

40. Zhu TH, Ding SJ, Li TT, Zhu LB, Huang XF, Zhang XM. Estrogen is an important mediator of mast cell activation in ovarian endometriomas. Reproduction. 2018;155:73-83.

41. Takamura M, Koga K, Izumi G, Urata Y, Nagai M, Hasegawa A, Harada M, Hirata T, Hirota Y, Wada-Hiraike O, et al. Neutrophil depletion reduces endometriotic lesion formation in mice. Am J Reprod Immunol. 2016;76:193-8.

42. Wang XM, Ma ZY, Song N. Inflammatory cytokines IL-6, IL-10, IL-13, TNFalpha and peritoneal fluid flora were associated with infertility in patients with endometriosis. Eur Rev Med Pharmacol Sci. 2018;22:2513-8.

43. Kolaczkowska E, Kubes P. Neutrophil recruitment and function in health and inflammation. Nat Rev Immunol. 2013;13:159-75.

44. Na YJ, Yang SH, Baek DW, Lee DH, Kim KH, Choi YM, Oh ST, Hong YS, Kwak $J Y$, Lee KS. Effects of peritoneal fluid from endometriosis patients on the release of vascular endothelial growth factor by neutrophils and monocytes. Hum Reprod. 2006;21:1846-55.

45. Saito S, Nakashima A, Ito M, Shima T. Clinical implication of recent advances in our understanding of IL-17 and reproductive immunology. Expert Rev Clin Immunol. 2011;7:649-57.

46. Gogacz M, Winkler I, Bojarska-Junak A, Tabarkiewicz J, Semczuk A, Rechberger T, Adamiak A. Increased percentage of Th17 cells in peritoneal fluid is associated with severity of endometriosis. J Reprod Immunol. 2016; 117:39-44.

47. Hirata T, Osuga Y, Takamura M, Saito A, Hasegawa A, Koga K, Yoshino O, Hirota Y, Harada M, Taketani Y. Interleukin-17F increases the secretion of interleukin-8 and the expression of cyclooxygenase 2 in endometriosis. Fertil Steril. 2011:96:113-7.

48. Garlanda C, Dinarello CA, Mantovani A. The interleukin-1 family: back to the future. Immunity. 2013:39:1003-18.

49. Sikora J, Ferrero S, Mielczarek-Palacz A, Kondera-Anasz Z. The delicate balance between the good and the bad IL-1 proinflammatory effects in endometriosis. Curr Med Chem. 2018;25:2105-21.

50. Jiang JF, Deng Y, Xue W, Zheng TP, Sun AJ. Increased expression of interleukin 37 in the eutopic and ectopic endometrium of patients with ovarian endometriosis. Reprod Sci. 2016;23:244-8.

51. Fan YY, Chen HY, Chen W, Liu YN, Fu Y, Wang LN. Expression of inflammatory cytokines in serum and peritoneal fluid from patients with different stages of endometriosis. Gynecol Endocrinol. 2018;34:507-12.

52. Jiang J, Yu K, Jiang Z, Xue M. IL-37 affects the occurrence and development of endometriosis by regulating the biological behavior of endometrial stromal cells through multiple signaling pathways. Biol Chem. 2018;399: 1325-37.

53. Conti P, Caraffa A, Mastrangelo F, Tettamanti L, Ronconi G, Frydas I, Kritas SK, Theoharides TC. Critical role of inflammatory mast cell in fibrosis: potential therapeutic effect of IL-37. Cell Prolif. 2018;51:e12475.

54. Xu WD, Zhao Y, Liu Y. Insights into IL-37, the role in autoimmune diseases. Autoimmun Rev. 2015;14:1170-5.

55. Kaabachi W, Kacem O, Belhaj R, Hamzaoui A, Hamzaoui K. Interleukin-37 in endometriosis. Immunol Lett. 2017;185:52-5.

56. Mari-Alexandre J, Barcelo-Molina M, Belmonte-Lopez E, Garcia-Oms J, Estelles A, Braza-Boils A, Gilabert-Estelles J. Micro-RNA profile and proteins in peritoneal fluid from women with endometriosis: their relationship with sterility. Fertil Steril. 2018;109:675-84.

57. Nematian SE, Mamillapalli R, Kadakia TS, Majidi ZM, Moustafa S, Taylor HS. Systemic inflammation induced by microRNAs: endometriosis-derived alterations in circulating microRNA $125 b-5 p$ and let-7b-5p regulate macrophage cytokine production. J Clin Endocrinol Metab. 2018;103:64-74.

58. Wang XQ, Zhou WJ, Luo XZ, Tao Y, Li DJ. Synergistic effect of regulatory $T$ cells and proinflammatory cytokines in angiogenesis in the endometriotic milieu. Hum Reprod. 2017;32:1304-17.

59. Wang WJ, Liu FJ, Xin-Liu, Hao CF, Bao HC, Qu QL, Liu XM. Adoptive transfer of pregnancy-induced CD4+CD25+ regulatory $T$ cells reverses the increase 
in abortion rate caused by interleukin 17 in the CBA/JxBALB/C mouse model. Hum Reprod. 2014;29:946-52.

60. Ahn SH, Edwards AK, Singh SS, Young SL, Lessey BA, Tayade C. IL-17A contributes to the pathogenesis of endometriosis by triggering Proinflammatory cytokines and angiogenic growth factors. J Immunol. 2015;195:2591-600.

61. Matson BC, Quinn KE, Lessey BA, Young SL, Caron KM. Elevated levels of adrenomedullin in eutopic endometrium and plasma from women with endometriosis. Fertil Steril. 2018;109:1072-8.

62. Volpato LK, Horewicz W, Bobinski F, Martins DF, Piovezan AP. Annexin A1, FPR2/ALX, and inflammatory cytokine expression in peritoneal endometriosis. J Reprod Immunol. 2018;129:30-5.

63. Soni UK, Chadchan SB, Kumar V, Ubba V, Khan M, Vinod B, Konwar R, Bora HK, Rath SK, Sharma S, Jha RK. A high level of TGF-B1 promotes endometriosis development via cell migration, adhesiveness, colonization, and invasivenessdagger. Biol Reprod. 2019:100:917-38.

64. Choi HJ, Chung TW, Choi HJ, Han JH, Choi JH, Kim CH, Ha KT. Increased alpha2-6 sialylation of endometrial cells contributes to the development of endometriosis. Exp Mol Med. 2018;50:164.

65. Howard FM. Endometriosis and mechanisms of pelvic pain. J Minim Invasive Gynecol. 2009;16:540-50.

66. Peng Y, Ma J, Lin J. Activation of the CXCL16/CXCR6 Axis by TNF-alpha contributes to ectopic endometrial stromal cells migration and invasion. Reprod Sci. 2019;26:420-7.

67. Arosh JA, Lee J, Balasubbramanian D, Stanley JA, Long CR, Meagher MW Osteen KG, Bruner-Tran KL, Burghardt RC, Starzinski-Powitz A, Banu SK. Molecular and preclinical basis to inhibit PGE2 receptors EP2 and EP4 as a novel nonsteroidal therapy for endometriosis. Proc Natl Acad Sci U S A. 2015;112:9716-21

68. Chang HM, Wu HC, Sun ZG, Lian F, Leung P. Neurotrophins and glial cell line-derived neurotrophic factor in the ovary: physiological and pathophysiological implications. Hum Reprod Update. 2019;25: 224-42.

69. Odagiri K, Konno R, Fujiwara H, Netsu S, Yang C, Suzuki M. Smooth muscle metaplasia and innervation in interstitium of endometriotic lesions related to pain. Fertil Steril. 2009;92:1525-31.

70. Mita S, Shimizu Y, Sato A, Notsu T, Imada K, Kyo S. Dienogest inhibits nerve growth factor expression induced by tumor necrosis factor-alpha or interleukin-1 beta. Fertil Steril. 2014;101:595-601.

71. Novella-Maestre E, Herraiz S, Vila-Vives JM, Carda C, Ruiz-Sauri A, Pellicer A. Effect of antiangiogenic treatment on peritoneal endometriosis-associated nerve fibers. Fertil Steril. 2012;98:1209-17.

72. McKinnon B, Bersinger NA, Wotzkow C, Mueller MD. Endometriosisassociated nerve fibers, peritoneal fluid cytokine concentrations, and pain in endometriotic lesions from different locations. Fertil Steril. 2012; 97:373-80.

73. Wang G, Tokushige N, Fraser IS. Nerve fibers and menstrual cycle in peritoneal endometriosis. Fertil Steril. 2011;95:2772-4.

74. Kamimura K, Inoue R, Nagoya T, Sakai N, Goto R, Ko M, Niwa Y, Terai S. Autonomic nervous system network and liver regeneration. World J Gastroenterol. 2018;24:1616-21.

75. Monica BM, Smith PG. Estrogen and female reproductive tract innervation: cellular and molecular mechanisms of autonomic neuroplasticity. Auton Neurosci. 2015;187:1-17.

76. Kaelberer MM, Buchanan KL, Klein ME, Barth BB, Montoya MM, Shen X, Bohorquez DV. A gut-brain neural circuit for nutrient sensory transduction. Science. 2018;361(6408):eaat5236.

77. Richeri A, Bianchimano P, Crutcher KA, Brauer MM. Reduced sympathetic neurite outgrowth on uterine tissue sections from rats treated with estrogen. Cell Tissue Res. 2010;340:287-301.

78. Liang Y, Yao S. Potential role of estrogen in maintaining the imbalanced sympathetic and sensory innervation in endometriosis. Mol Cell Endocrinol. 2016:424:42-9.

79. Possover M, Tersiev P, Angelov DN. Comparative study of the neuropeptide$Y$ sympathetic nerves in endometriotic involved and noninvolved sacrouterine ligaments in women with pelvic endometriosis. J Minim Invasive Gynecol. 2009;16:340-3.

80. Rob L, Halaska M, Robova H. Nerve-sparing and individually tailored surgery for cervical cancer. Lancet Oncol. 2010;11:292-301.

81. Hollabaugh RJ, Steiner MS, Sellers KD, Samm BJ, Dmochowski RR. Neuroanatomy of the pelvis: implications for colonic and rectal resection. Dis Colon Rectum. 2000;43:1390-7.
82. Shoja MM, Sharma A, Mirzayan N, Groat C, Watanabe K, Loukas M, Tubbs RS Neuroanatomy of the female abdominopelvic region: a review with application to pelvic pain syndromes. Clin Anat. 2013;26:66-76.

83. Ricu M, Paredes A, Greiner M, Ojeda SR, Lara HE. Functional development of the ovarian noradrenergic innervation. Endocrinology. 2008;149:50-6.

84. Zhu L, Huang Q, Huang X, Zhang J, Xu H, Zhang X. Decreased nerve fibers in the oviduct isthmus of women with endometriosis. Acta Histochem. 2014;116:871-7.

85. Tokushige N, Russell P, Black K, Barrera H, Dubinovsky S, Markham R, Fraser IS. Nerve fibers in ovarian endometriomas. Fertil Steril. 2010;94:1944-7.

86. Liang Y, Liu D, Yang F, Pan W, Zeng F, Wu J, Xie H, Li J, Yao S. Perineural invasion in endometriotic lesions contributes to endometriosis-associated pain. J Pain Res. 2018;11:1999-2009.

87. lanieri MM, Mautone D, Ceccaroni M. Recurrence in deep infiltrating endometriosis: a systematic review of the literature. J Minim Invasive Gynecol. 2018;25:786-93.

88. Kelm JA, Lancellotti CL, Donadio N, Auge AP, Lima SM, Aoki T, Ribeiro PA. Nerve fibers in uterosacral ligaments of women with deep infiltrating endometriosis. J Reprod Immunol. 2008;79:93-9.

89. A randomised controlled trial to assess the efficacy of laparoscopic uterosacral nerve ablation (LUNA) in the treatment of chronic pelvic pain: the trial protocol [ISRCTN41196151]. BMC Womens Health. 2003;3:6.

90. De Vadder F, Grasset E, Manneras HL, Karsenty G, Macpherson AJ, Olofsson LE, Backhed F. Gut microbiota regulates maturation of the adult enteric nervous system via enteric serotonin networks. Proc Natl Acad Sci U S A. 2018;115:6458-63.

91. Avetisyan M, Rood JE, Huerta LS, Sengupta R, Wright-Jin E, Dougherty JD, Behrens EM, Heuckeroth RO. Muscularis macrophage development in the absence of an enteric nervous system. Proc Natl Acad Sci U S A. 2018;115: 4696-701.

92. Duan L, Hope JM, Guo S, Ong Q, Francois A, Kaplan L, Scherrer G, Cui B. Optical activation of TrkA signaling. ACS Synth Biol. 2018;7:1685-93.

93. Peng B, Zhan H, Alotaibi F, Alkusayer GM, Bedaiwy MA, Yong PJ. Nerve growth factor is associated with sexual pain in women with endometriosis. Reprod Sci. 2018;25:540-9.

94. Merighi A, Salio C, Ghirri A, Lossi L, Ferrini F, Betelli C, Bardoni R. BDNF as a pain modulator. Prog Neurobiol. 2008;85:297-317.

95. Sikandar S, Minett MS, Millet Q, Santana-Varela S, Lau J, Wood JN, Zhao J. Brain-derived neurotrophic factor derived from sensory neurons plays a critical role in chronic pain. Brain. 2018;141:1028-39.

96. Ding S, Zhu T, Tian Y, Xu P, Chen Z, Huang X, Zhang X. Role of brainderived neurotrophic factor in endometriosis pain. Reprod Sci. 2018;25: 1045-57.

97. Wessels JM, Kay VR, Leyland NA, Agarwal SK, Foster WG. Assessing brainderived neurotrophic factor as a novel clinical marker of endometriosis. Fertil Steril. 2016;105:119-28.

98. Bonaz B, Sinniger $V$, Pellissier $S$. The vagus nerve in the neuro-immune axis: implications in the pathology of the gastrointestinal tract. Front Immunol. 2017:8:1452.

99. Liang Y, Wang W, Huang J, Tan H, Liu T, Shang C, Liu D, Guo L, Yao S. Potential role of Semaphorin $3 \mathrm{~A}$ and its receptors in regulating aberrant sympathetic innervation in peritoneal and deep infiltrating endometriosis. PLoS One. 2015;10:e146027.

100. Bonaz B, Sinniger V, Pellissier S. Vagus nerve stimulation: a new promising therapeutic tool in inflammatory bowel disease. J Intern Med. 2017;282:46-63.

101. Willemze RA, Welting $O$, van Hamersveld P, Verseijden C, Nijhuis LE, Hilbers FW, Meijer SL, Heesters BA, Folgering J, Darwinkel $H$, et al. Loss of intestinal sympathetic innervation elicits an innate immune driven colitis. Mol Med. 2019:25:1.

102. Straub RH, Grum F, Strauch U, Capellino S, Bataille F, Bleich A, Falk W, Scholmerich J, Obermeier F. Anti-inflammatory role of sympathetic nerves in chronic intestinal inflammation. Gut. 2008;57:911-21.

103. Kunath J, Delaroque N, Szardenings M, Neundorf I, Straub RH. Sympathetic nerve repulsion inhibited by designer molecules in vitro and role in experimental arthritis. Life Sci. 2017;168:47-53.

104. Taborsky GJ, Mei Q, Hackney DJ, Figlewicz DP, LeBoeuf R, Mundinger TO. Loss of islet sympathetic nerves and impairment of glucagon secretion in the NOD mouse: relationship to invasive insulitis. Diabetologia. 2009;52: 2602-11.

105. Nance DM, Sanders VM. Autonomic innervation and regulation of the immune system (1987-2007). Brain Behav Immun. 2007;21:736-45. 
106. Pongratz G, Straub $\mathrm{RH}$. The sympathetic nervous response in inflammation. Arthritis Res Ther. 2014;16:504.

107. Lee KK, Jharap B, Maser EA, Colombel JF. Impact of concomitant endometriosis on phenotype and natural history of inflammatory bowel disease. Inflamm Bowel Dis. 2016;22:159-63.

108. Ganesh T, Banik A, Dingledine R, Wang W, Amaradhi R. Peripherally restricted, highly potent, selective, aqueous-soluble EP2 antagonist with anti-inflammatory properties. Mol Pharm. 2018;15:5809-17.

109. Ribeiro-da-Silva M, Vasconcelos DM, Alencastre IS, Oliveira MJ, Linhares D, Neves N, Costa G, Henrique R, Lamghari M, Alves CJ. Interplay between sympathetic nervous system and inflammation in aseptic loosening of hip joint replacement. Sci Rep. 2018;8:16044.

110. Weidler C, Holzer C, Harbuz M, Hofbauer R, Angele P, Scholmerich J, Straub $\mathrm{RH}$. Low density of sympathetic nerve fibres and increased density of brain derived neurotrophic factor positive cells in RA synovium. Ann Rheum Dis. 2005;64:13-20

111. Imdad A, Nicholson MR, Tanner-Smith EE, Zackular JP, Gomez-Duarte OG, Beaulieu DB, Acra S. Fecal transplantation for treatment of inflammatory bowel disease. Cochrane Database Syst Rev. 2018;11:D12774.

112. Bai A, Lu N, Guo Y, Chen J, Liu Z. Modulation of inflammatory response via alpha2-adrenoceptor blockade in acute murine colitis. Clin Exp Immunol. 2009;156:353-62.

113. Di Giovangiulio M, Verheijden S, Bosmans G, Stakenborg N, Boeckxstaens GE, Matteoli $G$. The neuromodulation of the intestinal immune system and its relevance in inflammatory bowel disease. Front Immunol. 2015;6:590.

114. Moynes DM, Lucas GH, Beyak MJ, Lomax AE. Effects of inflammation on the innervation of the colon. Toxicol Pathol. 2014:42:111-7.

115. Rubio A, Pellissier S, Picot A, Dantzer C, Bonaz B. The link between negative affect, vagal tone, and visceral sensitivity in quiescent Crohn's disease. Neurogastroenterol Motil. 2014;26:1200-3.

116. Pellissier S, Dantzer C, Canini F, Mathieu N, Bonaz B. Psychological adjustment and autonomic disturbances in inflammatory bowel diseases and irritable bowel syndrome. Psychoneuroendocrino. 2010;35:653-62.

117. Brzozowski B, Mazur-Bialy A, Pajdo R, Kwiecien S, Bilski J, Zwolinska-Wcislo $M$, Mach T, Brzozowski T. Mechanisms by which stress affects the experimental and clinical inflammatory bowel disease (IBD): role of braingut Axis. Curr Neuropharmacol. 2016;14:892-900.

118. Pellissier S, Bonaz B. The place of stress and emotions in the irritable bowel syndrome. Vitam Horm. 2017;103:327-54.

119. Engel T, Ben-Horin S, Beer-Gabel M. Autonomic dysfunction correlates with clinical and inflammatory activity in patients with Crohn's disease. Inflamm Bowel Dis. 2015;21:2320-6.

120. Bonaz B, Bazin T, Pellissier S. The Vagus nerve at the Interface of the microbiota-gut-brain Axis. Front Neurosci. 2018;12:49.

121. Boisse L, Chisholm SP, Lukewich MK, Lomax AE. Clinical and experimental evidence of sympathetic neural dysfunction during inflammatory bowel disease. Clin Exp Pharmacol Physiol. 2009;36:1026-33.

122. Straub RH, Wiest R, Strauch UG, Harle P, Scholmerich J. The role of the sympathetic nervous system in intestinal inflammation. Gut. 2006;55:1640-9.

123. Weatherby KE, Zwilling BS, Lafuse WP. Resistance of macrophages to Mycobacterium avium is induced by alpha2-adrenergic stimulation. Infect Immun. 2003;71:22-9.

124. Willemze RA, Luyer MD, Buurman WA, de Jonge WJ. Neural reflex pathways in intestinal inflammation: hypotheses to viable therapy. Nat Rev Gastroenterol Hepatol. 2015;12:353-62.

125. Noble BT, Brennan FH, Popovich PG. The spleen as a neuroimmune interface after spinal cord injury. J Neuroimmunol. 2018;321:1-11.

126. Murray K, Godinez DR, Brust-Mascher I, Miller EN, Gareau MG, Reardon C. Neuroanatomy of the spleen: mapping the relationship between sympathetic neurons and lymphocytes. PLoS One. 2017;12:e182416.

127. Jess T, Frisch M, Jorgensen KT, Pedersen BV, Nielsen NM. Increased risk of inflammatory bowel disease in women with endometriosis: a nationwide Danish cohort study. Gut. 2012;61:1279-83.

128. Bonaz B, Sinniger V, Pellissier S. Anti-inflammatory properties of the vagus nerve: potential therapeutic implications of vagus nerve stimulation. J Physiol. 2016:594:5781-90.

129. Spiller R, Major G. IBS and IBD_separate entities or on a spectrum? Nat Rev Gastroenterol Hepatol. 2016;13:613-21.

130. Guerrero-Alba R, Valdez-Morales EE, Jimenez-Vargas NN, Lopez-Lopez C, Jaramillo-Polanco J, Okamoto T, Nasser Y, Bunnett NW, Lomax AE, Vanner
SJ. Stress activates pronociceptive endogenous opioid signalling in DRG neurons during chronic colitis. Gut. 2017;66:2121-31.

131. Herrmann M, Anders S, Straub RH, Jenei-Lanzl Z. TNF inhibits catecholamine production from induced sympathetic neuron-like cells in rheumatoid arthritis and osteoarthritis in vitro. Sci Rep. 2018;8:9645.

132. Koopman FA, Tang MW, Vermeij J, de Hair MJ, Choi IY, Vervoordeldonk MJ, Gerlag DM, Karemaker JM, Tak PP. Autonomic dysfunction precedes development of rheumatoid arthritis: a prospective cohort study. Ebiomedicine. 2016;6:231-7.

133. Jenei-Lanzl Z, Capellino S, Kees F, Fleck M, Lowin T, Straub RH. Antiinflammatory effects of cell-based therapy with tyrosine hydroxylasepositive catecholaminergic cells in experimental arthritis. Ann Rheum Dis. 2015;74:444-51.

134. Bellinger DL, Lorton D. Sympathetic nerve hyperactivity in the spleen: causal for nonpathogenic-driven chronic immune-mediated inflammatory diseases (IMIDs)? Int J Mol Sci. 2018;19(4):1188.

135. Liu Y, Rui XX, Shi H, Qiu YH, Peng YP. Norepinephrine inhibits Th17 cells via beta2-adrenergic receptor (beta2-AR) signaling in a mouse model of rheumatoid arthritis. Med Sci Monit. 2018;24:1196-204.

136. Nackley AG, Tan KS, Fecho K, Flood P, Diatchenko L, Maixner W. Catechol-Omethyltransferase inhibition increases pain sensitivity through activation of both beta2- and beta3-adrenergic receptors. Pain. 2007;128:199-208.

137. Harle P, Mobius D, Carr DJ, Scholmerich J, Straub RH. An opposing timedependent immune-modulating effect of the sympathetic nervous system conferred by altering the cytokine profile in the local lymph nodes and spleen of mice with type II collagen-induced arthritis. Arthritis Rheum. 2005; 52:1305-13.

138. Chen P, Wang DB, Liang YM. Evaluation of estrogen in endometriosis patients: regulation of GATA-3 in endometrial cells and effects on Th2 cytokines. J Obstet Gynaecol Res. 2016;42:669-77.

139. Greaves E, Temp J, Esnal-Zufiurre A, Mechsner S, Horne AW, Saunders PT. Estradiol is a critical mediator of macrophage-nerve cross talk in peritoneal endometriosis. Am J Pathol. 2015;185:2286-97.

140. Gou Y, Li X, Li P, Zhang H, Xu T, Wang H, Wang B, Ma X, Jiang X, Zhang Z. Estrogen receptor beta upregulates CCL2 via NF-kappaB signaling in endometriotic stromal cells and recruits macrophages to promote the pathogenesis of endometriosis. Hum Reprod. 2019;34:646-58.

141. Krizsan-Agbas D, Pedchenko T, Hasan W, Smith PG. Oestrogen regulates sympathetic neurite outgrowth by modulating brain derived neurotrophic factor synthesis and release by the rodent uterus. Eur J Neurosci. 2003;18: 2760-8.

142. Barcena DAM, Oldeweme J, Arnold J, Schneider A, Mechsner S. Remodeling of estrogen-dependent sympathetic nerve fibers seems to be disturbed in adenomyosis. Fertil Steril. 2013;100:801-9.

143. Martinez GF, Bianchimano P, Brauer MM. Estrogen-induced collagen reorientation correlates with sympathetic denervation of the rat myometrium. Auton Neurosci. 2016;201:32-9.

144. Jana B, Meller KA, Czajkowska M, Calka J. Long-term estradiol-17beta exposure decreases the cholinergic innervation pattern of the pig ovary. Ann Anat. 2018;216:135-41.

145. Liang $Y$, Xie $H, W u$ J, Liu D, Yao S. Villainous role of estrogen in macrophage-nerve interaction in endometriosis. Reprod Biol Endocrinol. 2018;16:122.

146. Rafique S, Decherney AH. Medical management of endometriosis. Clin Obstet Gynecol. 2017;60:485-96.

147. Brown J, Crawford TJ, Allen C, Hopewell S, Prentice A. Nonsteroidal antiinflammatory drugs for pain in women with endometriosis. Cochrane Database Syst Rev. 2017;1:D4753.

148. Baurle S, Nagel J, Peters O, Brauer N, Ter Laak A, Preusse C, Rottmann A, Heldmann D, Bothe $U$, Blume $T$, et al. Identification of a Benzimidazolecarboxylic acid derivative (BAY 1316957) as a potent and selective human prostaglandin E2 receptor subtype 4 (hEP4-R) antagonist for the treatment of endometriosis. J Med Chem. 2019;62:2541-63.

149. Vercellini P, Vigano P, Somigliana E, Fedele L. Endometriosis: pathogenesis and treatment. Nat Rev Endocrinol. 2014;10:261-75.

150. Vercellini P, Buggio L, Berlanda N, Barbara G, Somigliana E, Bosari S. Estrogen-progestins and progestins for the management of endometriosis. Fertil Steril. 2016;106:1552-71.

151. Berlanda N, Somigliana E, Frattaruolo MP, Buggio L, Dridi D, Vercellini P. Surgery versus hormonal therapy for deep endometriosis: is it a choice of the physician? Eur J Obstet Gynecol Reprod Biol. 2017;209:67-71. 
152. Lang J, Yu Q, Zhang S, Li H, Gude K, von Ludwig C, Ren X, Dong L. Dienogest for treatment of endometriosis in Chinese women: a placebocontrolled, randomized, double-blind phase 3 study. J Women's Health (Larchmt). 2018;27:148-55.

153. Andres MP, Lopes LA, Baracat EC, Podgaec S. Dienogest in the treatment of endometriosis: systematic review. Arch Gynecol Obstet. 2015;292:523-9.

154. Tarjanne S, Ng C, Manconi F, Arola J, Mentula M, Maneck B, Fraser IS, Heikinheimo $\mathrm{O}$. Use of hormonal therapy is associated with reduced nerve fiber density in deep infiltrating, rectovaginal endometriosis. Acta Obstet Gynecol Scand. 2015;94:693-700.

155. Liao Z, Smith PG. Persistent genital hyperinnervation following progesterone administration to adolescent female rats. Biol Reprod. 2014;91:144.

156. Dull AM, Moga MA, Dimienescu OG, Sechel G, Burtea V, Anastasiu CV. Therapeutic approaches of resveratrol on endometriosis via antiinflammatory and anti-Angiogenic pathways. Molecules. 2019:24(4):667.

157. Vercellini P, Frattaruolo MP, Rosati R, Dridi D, Roberto A, Mosconi P, De Giorgi O, Cribiu FM, Somigliana E. Medical treatment or surgery for colorectal endometriosis? Results of a shared decision-making approach Hum Reprod. 2018;33:202-11.

158. Wang Q, Sun X. Recent advances in nanomedicines for the treatment of rheumatoid arthritis. Biomater Sci. 2017;5:1407-20.

159. Goenka L, George M, Sen M. A peek into the drug development scenario of endometriosis - a systematic review. Biomed Pharmacother. 2017;90:575-85.

160. Pazhohan A, Amidi F, Akbari-Asbagh F, Seyedrezazadeh E, Aftabi Y, Abdolalizadeh J, Khodarahmian M, Khanlarkhani N, Sobhani A. Expression and shedding of CD44 in the endometrium of women with endometriosis and modulating effects of vitamin D: a randomized exploratory trial. J Steroid Biochem Mol Biol. 2018;178:150-8.

161. Hasegawa A, Yoshino O, Osuga Y, Hirata T, Yano T, Taketani Y. High soluble CD44 concentration in peritoneal fluid in endometriosis. Fertil Steril. 2008; 89:1267-8.

162. Koushik S, Joshi N, Nagaraju S, Mahmood S, Mudeenahally K, Padmavathy R, Jegatheesan SK, Mullangi R, Rajagopal S. PAD4: pathophysiology, current therapeutics and future perspective in rheumatoid arthritis. Expert Opin Ther Targets. 2017;21:433-47.

163. Cheung TT, McInnes IB. Future therapeutic targets in rheumatoid arthritis? Semin Immunopathol. 2017:39:487-500

164. Liang Y, Wu J, Wang W, Xie H, Yao S. Pro-endometriotic niche in endometriosis. Reprod BioMed Online. 2019;38:549-59.

\section{Publisher's Note}

Springer Nature remains neutral with regard to jurisdictional claims in published maps and institutional affiliations.

Ready to submit your research? Choose BMC and benefit from:

- fast, convenient online submission

- thorough peer review by experienced researchers in your field

- rapid publication on acceptance

- support for research data, including large and complex data types

- gold Open Access which fosters wider collaboration and increased citations

- maximum visibility for your research: over $100 \mathrm{M}$ website views per year

At $\mathrm{BMC}$, research is always in progress.

Learn more biomedcentral.com/submissions 